\title{
Effect of Stacking Interactions on the Translation of Structurally Related Bis-thiosemicarbazone Ni(II) HER Catalysts to Modified Electrode Surfaces
}

Alexander J. Gupta, ${ }^{1}{ }^{1}$ Nicholas S. Vishnosky, ${ }^{2}$ Oleksandr Hietsoi, ${ }^{2}$ Yaroslav Losovyj, ${ }^{3}$ Jacob Strain, ${ }^{2}$ Joshua Spurgeon, ${ }^{4}$ Mark S. Mashuta, ${ }^{2}$ Rahul Jain, ${ }^{2}$

Robert M. Buchanan, ${ }^{2}$ Gautam Gupta, ${ }^{* 1}$ and Craig A. Grapperhaus ${ }^{* 2}$

${ }^{1}$ Department of Chemical Engineering, University of Louisville, Louisville, Kentucky 40292, USA.

${ }^{2}$ Department of Chemistry, University of Louisville, Louisville, Kentucky 40292, USA.

${ }^{3}$ Department of Chemistry, Indiana University Bloomington, Bloomington, Indiana, 47405, USA.

${ }^{4}$ Conn Center for Renewable Energy Research, University of Louisville, Louisville, Kentucky 40292, USA.

Table of Contents

Figure S1. ${ }^{1} \mathrm{H}$ NMR (400 MHz) of 2 in DMSO-d 6 ............................................................ 3

Figure S2. ${ }^{1} \mathrm{H}$ NMR (400 MHz) of 3 in DMSO-d 6 ........................................................... 4

Figure S3. FTIR of 2 as a powder (ATR). ................................................................. 4

Figure S4. FTIR of 3 as a powder (ATR). ................................................................. 5

Figure S5. UV-visible spectra of $1-3$ in acetonitrile. .................................................. 5

Table S1: Crystal data and structural refinement for Complexes 2 and 3. ............................ 6

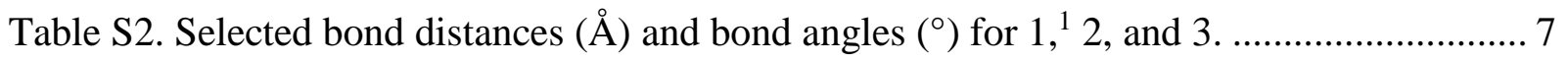

Table S3. Bond distances and angles of complex 2.......................................................... 8

Table S4. Bond distances and angles of complex 3........................................................... 9

Figure S6. Effects of reductive cycling from 0 to $-0.8 \mathrm{~V}$ vs. RHE at $50 \mathrm{mV} / \mathrm{s}$ on the performance of the three modified electrodes. Vertical scale is expanded to show peak cathodic current for each electrode. 10

Figure S7. Effect on HER performance of dipping modified electrodes in acetonitrile for 30

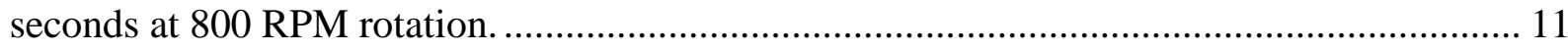

Figure S8. UV-Visible Spectroscopy data for complex 2 in solution.................................. 12

Figure S9. Raman spectra of various electrodes and organic solutions with bands labelled.... 12

Figure S10. Raman spectra of various electrodes and organic solutions with bands labelled.. 13

Figure S11. EDS of GC-2 before reductive cycling. ....................................................... 14

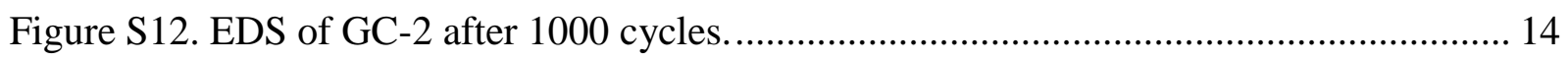

Figure S13. Survey scans of the GC-2 electrode before (red) and after (black) conditioning to

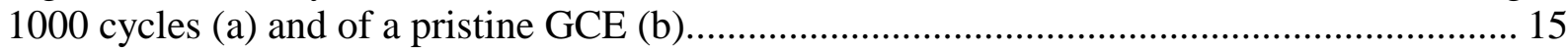


Table S5. Atomic \% of Ni2p, S2p and Ni1s before and after conditioning (1000 cycles)....... 15

Table S6. XPS fitting parameters for nickel (Figure 8A). ................................................... 16

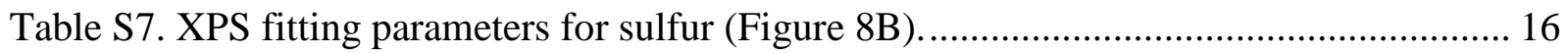

Table S8. XPS fitting parameters for nickel (Figure 10A). ................................................. 16

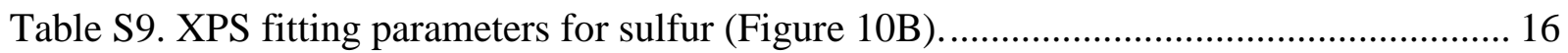

Figure S14. Elemental mapping of nickel and sulfur. ..................................................... 17

Figure S15. Equivalent circuit model used to fit EIS data................................................ 17

Table S10. Parameters obtained by fitting frequency response analysis (FRA) data to simulated

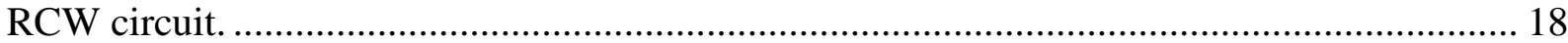

Figure S16. Nyquist plots showing the effects of reductive cycling from 0 to $-0.8 \mathrm{~V}$ vs. RHE at

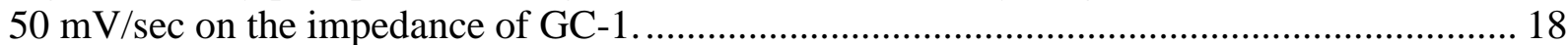

Figure S17. Nyquist plots showing the effects of reductive cycling from 0 to $-0.8 \mathrm{~V}$ vs. RHE at

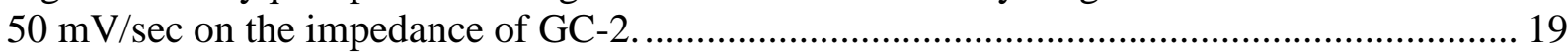

Figure S18. Nyquist plots showing the effects of cycling from 0 to $-0.8 \mathrm{~V}$ vs. RHE at $50 \mathrm{mV} / \mathrm{sec}$

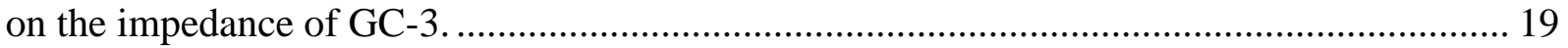

Figure S19. Cyclic voltammetry plots showing the capacitive effects of varying scan speed over a non-Faradaic potential range for GC $1-3$ as-deposited................................................. 20

Figure S20. Cyclic voltammetry plots showing the capacitive effects of varying scan speed over a non-Faradaic potential range for GC $1-3$ after cycling to peak activity.......................... 21

Figure S21. Plot of average current density at $0.3 \mathrm{~V}$ vs. RHE as measured during anodic sweep as a function of scan rate for as-deposited electrodes.................................................... 21

Figure S22. Plot of average current density at $0.3 \mathrm{~V}$ vs. RHE as measured during anodic sweep as a function of scan rate for GC $1-3$ after cycling to peak activity.................................. 22

Figure S23. Oxidation (top peak) and reduction (bottom peak) of ferricyanide redox couple at 5

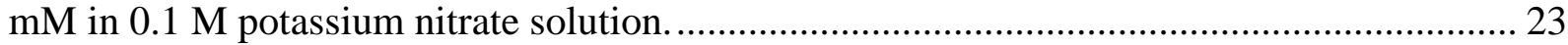

Figure S24. Plot of maximum current (top, for anodic sweeps) and minimum current (bottom, for cathodic sweeps) associated with ferricyanide reduction/oxidation cyclic voltammetry experiment as a function of the square root of scan rate....................................................... 24

Table S11. Slopes of the traces of maximum/minimum current densities as a function of the square root of scan rate for ferricyanide oxidation/reduction experiments as well as areas calculated from the Randles-Sevcik equation. 24

Figure S25. Plot of theoretical hydrogen, measured hydrogen, and Faradaic efficiencies for GC

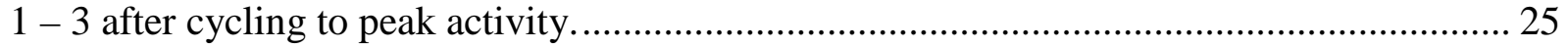

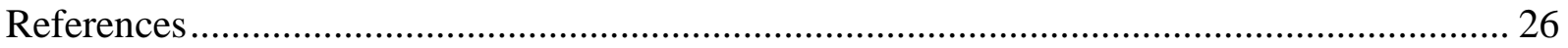




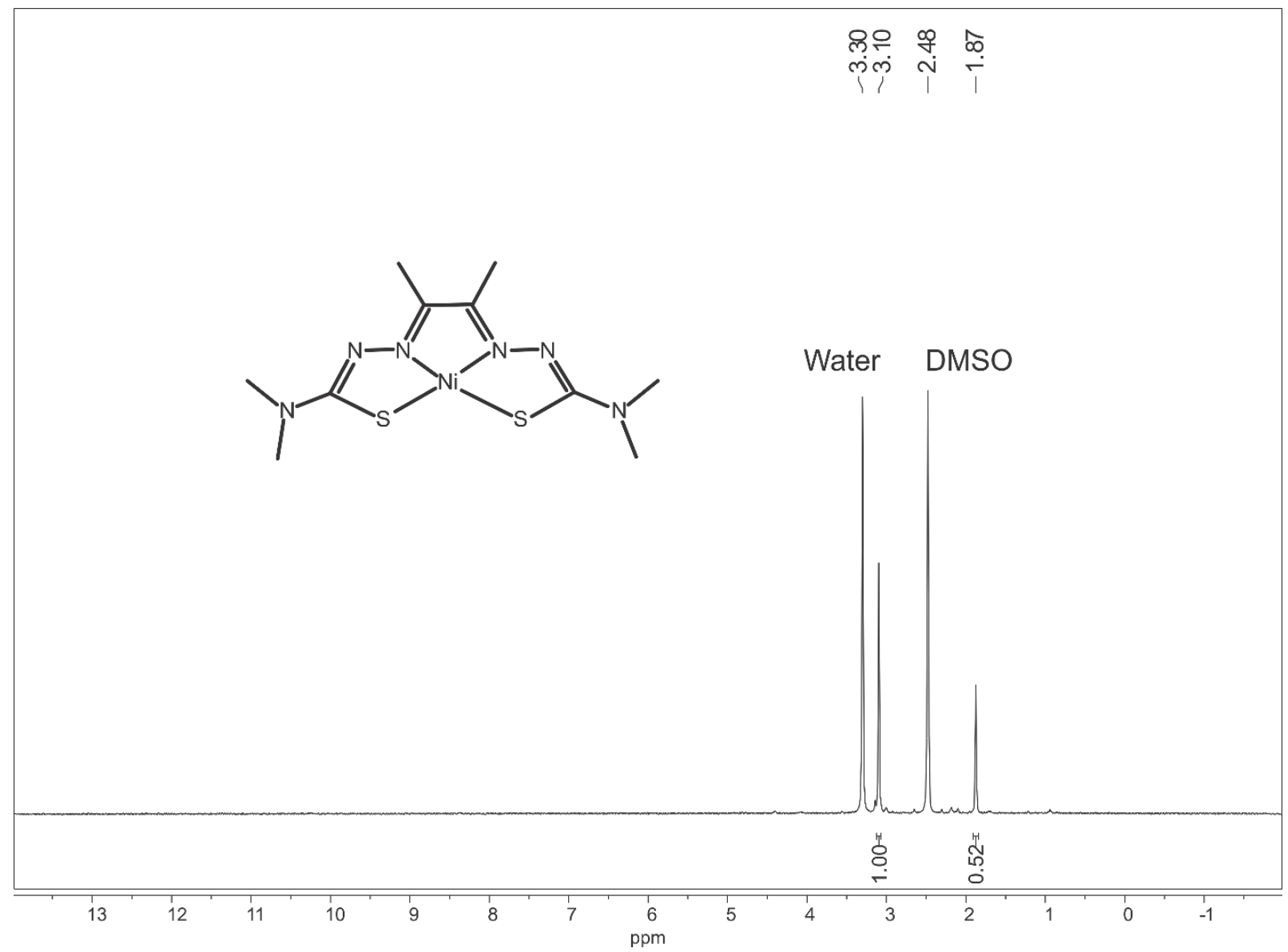

Figure S1. ${ }^{1} \mathrm{H}$ NMR (400 MHz) of 2 in DMSO-d6.

$\delta 1.87(\mathrm{~s}, 6 \mathrm{H}) 3.10(\mathrm{~s}, 12 \mathrm{H})$ 


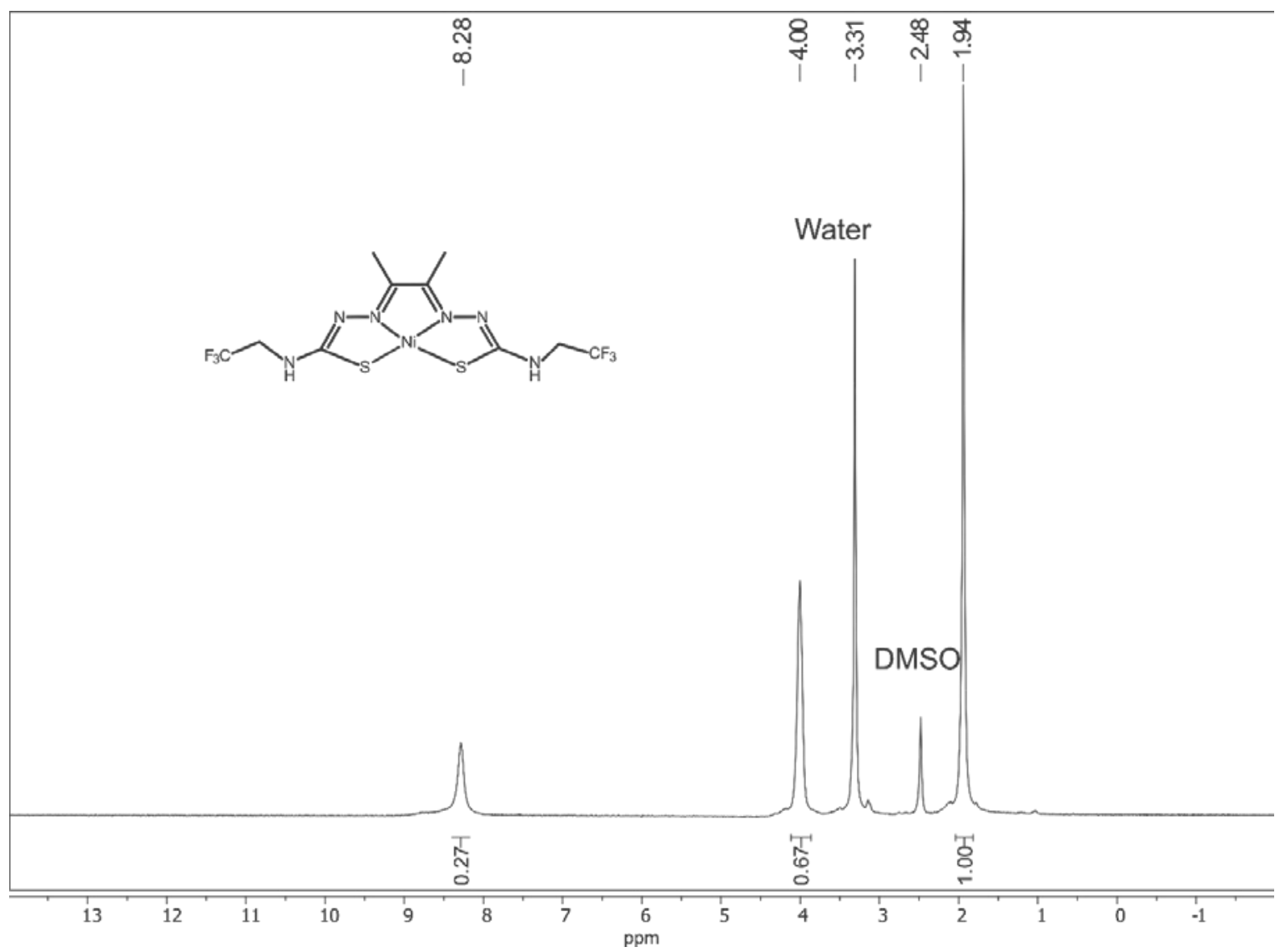

Figure S2. ${ }^{1} \mathrm{H}$ NMR (400 MHz) of 3 in DMSO-d6.

$\delta 1.94$ (s, 6H) 4.00 (br, 4H) 8.28 (s, 2H)

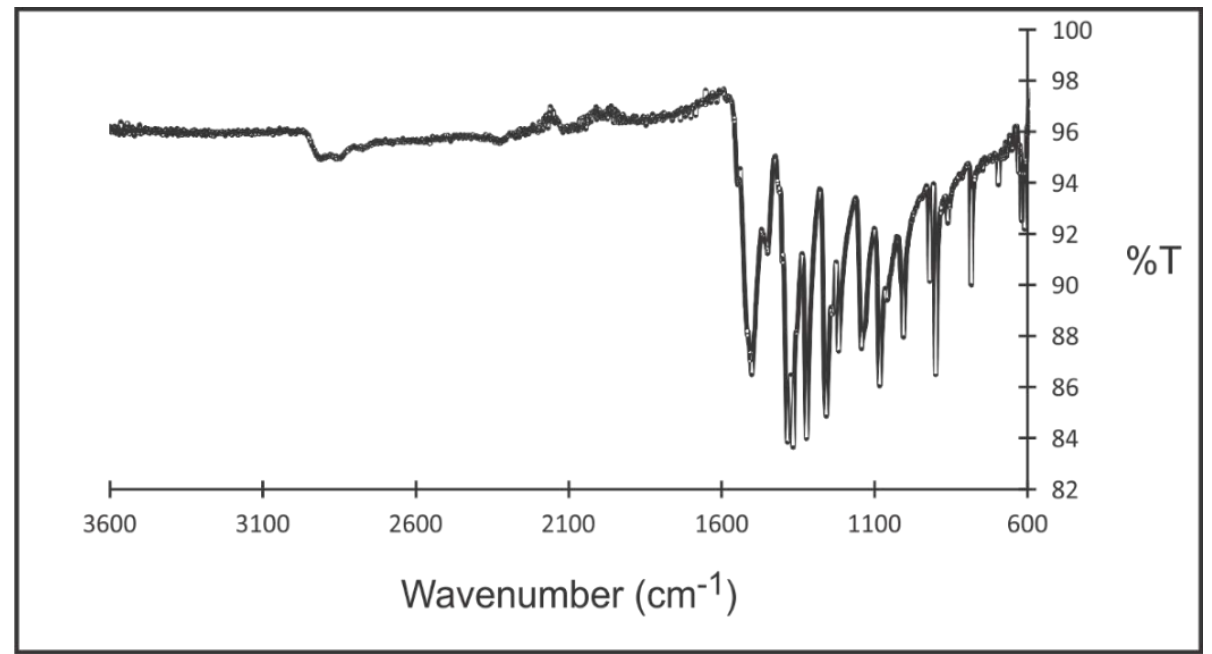

Figure S3. FTIR of 2 as a powder (ATR). 


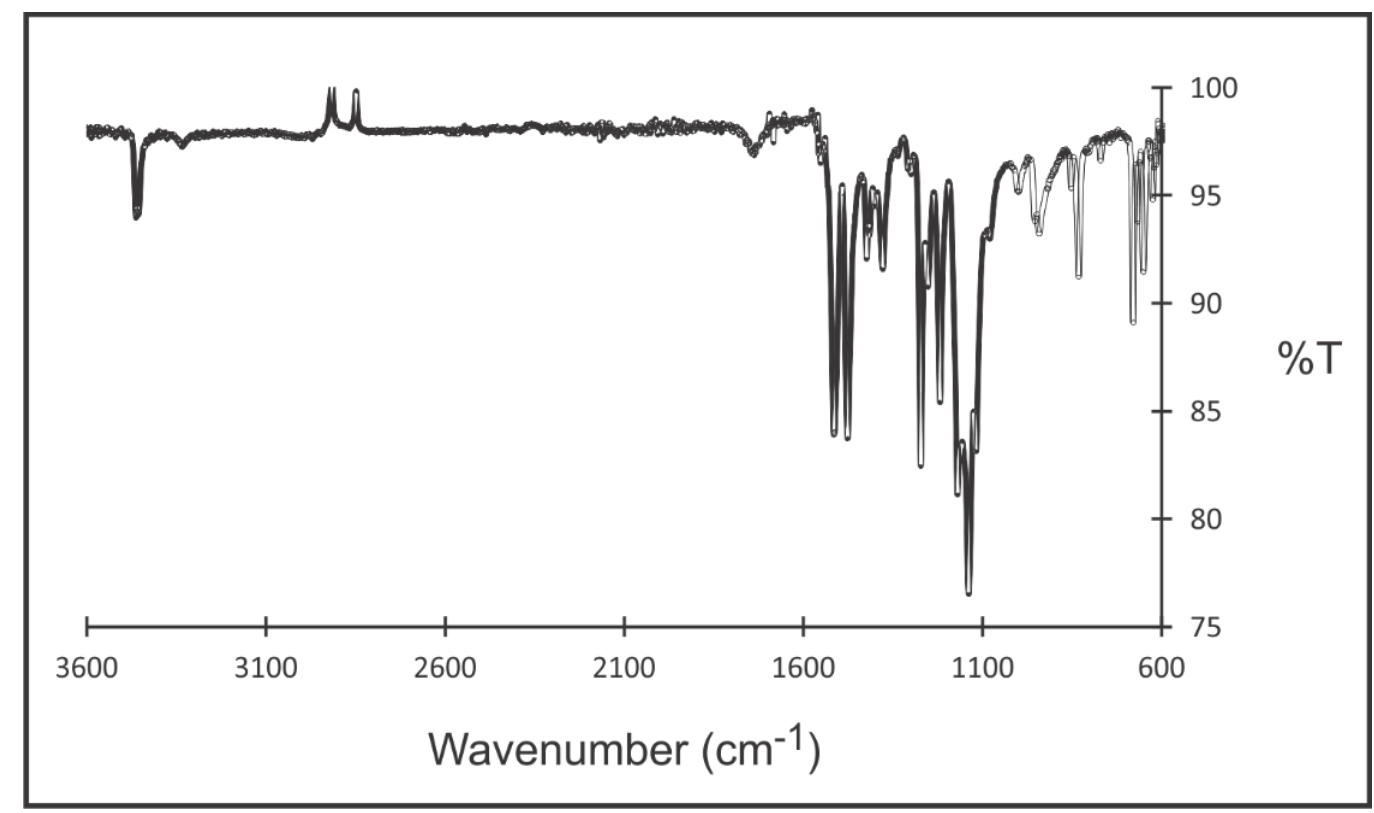

Figure S4. FTIR of 3 as a powder (ATR).

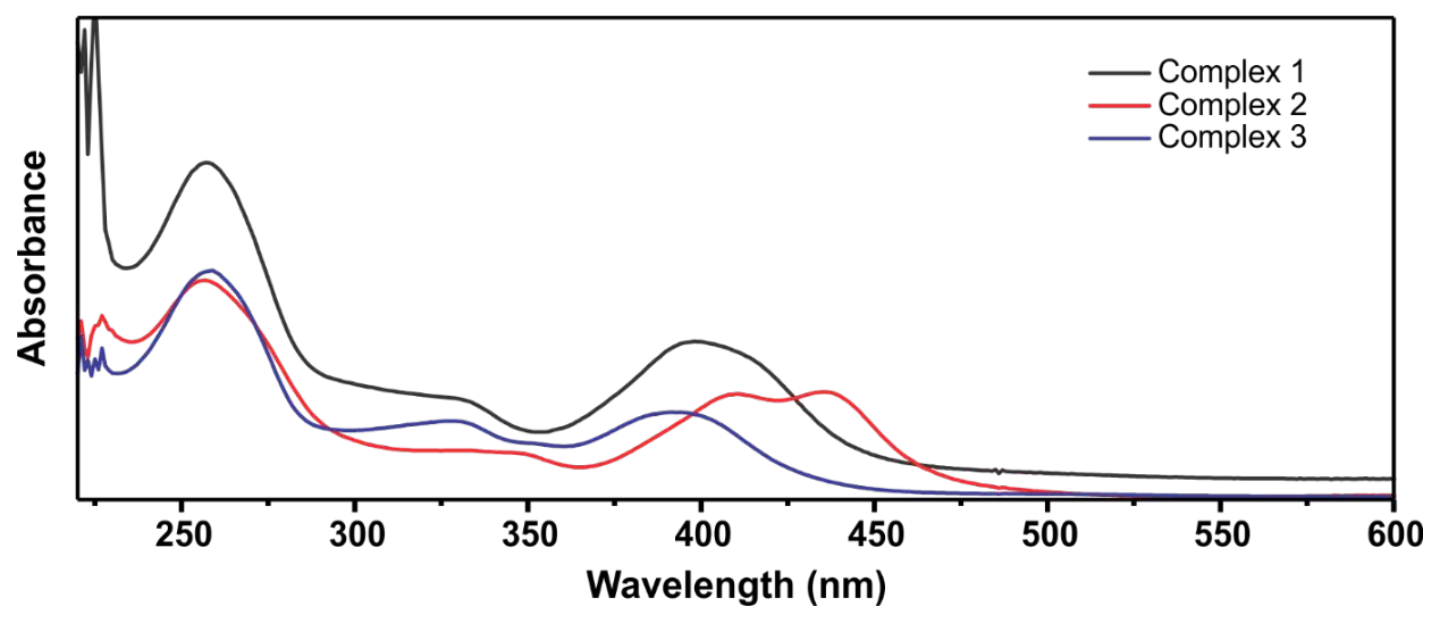

Figure S5. UV-visible spectra of $1-3$ in acetonitrile. 


\section{Table S1: Crystal data and structural refinement for Complexes 2 and 3.}

\begin{tabular}{|c|c|c|}
\hline Compound & NiATSDM (Complex 2) & NiATSM-F 6 (Complex 3) \\
\hline Empirical formula & $\mathrm{C}_{10} \mathrm{H}_{18} \mathrm{~N}_{6} \mathrm{NiS}_{2}$ & $\mathrm{C}_{10} \mathrm{H}_{12} \mathrm{~F}_{6} \mathrm{~N}_{6} \mathrm{NiS}_{2}$ \\
\hline Formula weight & 345.13 & 453.09 \\
\hline Temperature (K) & $100.2(6)$ & $100(2)$ \\
\hline Wavelength $(\AA)$ & 0.71073 & 0.71073 \\
\hline Crystal system & Triclinic & Triclinic \\
\hline Space group & $\mathrm{P}-1$ & $\mathrm{P}-1$ \\
\hline \multirow[t]{6}{*}{ Unit cell dimension } & $\mathrm{a}=7.33437(16)$ & $\mathrm{a}=8.3598(10)$ \\
\hline & $\mathrm{b}=8.5595(2)$ & $b=9.4027(10)$ \\
\hline & $c=11.6784$ & $c=13.5112(17)$ \\
\hline & $\alpha=103.865(2)$ & $\alpha=109.467$ \\
\hline & $\beta=98.2102(19)$ & $\beta=105.475(11)$ \\
\hline & $\gamma=91.7205(18)$ & $\gamma=91.382(9)$ \\
\hline Volume $\left(\AA^{3}\right)$ & 702.92(3) & 957.53(19) \\
\hline $\mathrm{Z}$ & 2 & 2 \\
\hline$\rho_{\text {calcd.. }}\left(\mathrm{Mg} / \mathrm{m}^{3}\right)$ & 1.631 & 1.571 \\
\hline Abs. coeff. $\left(\mathrm{mm}^{-1}\right)$ & 1.672 & 1.291 \\
\hline $\mathrm{F}(000)$ & 360 & 456 \\
\hline Crystal colour, habit & Orange prism & Red-brown plate \\
\hline Crystal size $\left(\mathrm{mm}^{3}\right)$ & $0.40 \times 0.34 \times 0.18$ & $0.41 \times 0.08 \times 0.01$ \\
\hline$\Theta$ range data collection $\left(^{\circ}\right)$ & 3.40 to 31.57 & 3.30 to 26.67 \\
\hline \multirow[t]{3}{*}{ Index ranges } & $-10 \leq \mathrm{h} \leq 10$ & $-10 \leq \mathrm{h} \leq 10$ \\
\hline & $-12 \leq \mathrm{k} \leq 12$ & $-11 \leq \mathrm{k} \leq 11$ \\
\hline & $-17 \leq 1 \leq 17$ & $-17 \leq 1 \leq 17$ \\
\hline Reflections collected & 19998 & 13904 \\
\hline Independent reflections & $4702[\mathrm{R}(\mathrm{int})=0.0217]$ & $4031[\mathrm{R}(\mathrm{int})=0.058]$ \\
\hline Completeness to $\theta$ max & 99.9 \% & $99.7 \%$ \\
\hline Absorption correction & Multiscan & Multiscan \\
\hline Max. and min. trans. & 1.000 and 0.778 & 1.00 and 0.678 \\
\hline Refinement method & Full-matrix least-squares on $\mathrm{F}^{2}$ & Full-matrix least-squares on $\mathrm{F}^{2}$ \\
\hline Data/restraints/params & $4702 / 0 / 244$ & $4031 / 0 / 288$ \\
\hline Goodness-of-fit- on $\mathrm{F}^{2}$ & 1.013 & 1.074 \\
\hline Final $\mathrm{R}$ indices & $\mathrm{R} 1=0.0196$ & $\mathrm{R} 1=0.0500$ \\
\hline$[\mathrm{I}>2 \sigma(\mathrm{I})]^{\mathrm{a}, \mathrm{b}}$ & $\mathrm{wR} 2=0.0533$ & $\mathrm{wR} 2=0.1099$ \\
\hline \multirow[t]{2}{*}{$\mathrm{R}$ indices (all data) } & $\mathrm{R} 1=0.0211$ & $\mathrm{R} 1=0.0647$ \\
\hline & $\mathrm{wR} 2=0.0545$ & $\mathrm{wR} 2=0.1188$ \\
\hline $\begin{array}{l}\text { Largest difference peak and hole } \\
\left(\mathrm{e} \cdot \AA^{3}\right)\end{array}$ & 0.525 and -0.285 & 1.176 and -0.477 \\
\hline
\end{tabular}


Table S2. Selected bond distances $(\AA)$ and bond angles $\left(^{\circ}\right)$ for 1,1 , and 3.

\begin{tabular}{|l|l|l|l|}
\hline Bond distance & $\mathbf{1}^{\mathrm{a}}$ & $\mathbf{2}$ & $\mathbf{3}$ \\
\hline Ni-N1 & $1.861(2)$ & $1.8528(8)$ & $1.854(3)$ \\
\hline Ni-N3 & $1.855(2)$ & $1.8580(8)$ & $1.863(3)$ \\
\hline Ni-S1 & $2.1546(6)$ & $2.1617(3)$ & $2.1542(11)$ \\
\hline Ni-S2 & $2.1598(7)$ & $2.1576(2)$ & $2.1527(10)$ \\
\hline S1-C5 & $1.767(3)$ & $1.7665(9)$ & $1.763(4)$ \\
\hline N2-C5 & $1.315(3)$ & $1.3222(12)$ & $1.308(5)$ \\
\hline N1-N2 & $1.378(3)$ & $1.3694(11)$ & $1.381(4)$ \\
\hline N1-C1 & $1.303(3)$ & $1.3038(11)$ & $1.310(5)$ \\
\hline C1-C3 & $1.489(4)$ & $1.4857(13)$ & $1.487(5)$ \\
\hline C1-C2 & $1.470(3)$ & $1.4658(13)$ & $1.474(5)$ \\
\hline N5-C5 & $1.339(3)$ & $1.3456(12)$ & $1.350(5)$ \\
\hline N5-C7 & $1.449(3)$ & $1.4570(13)$ & $1.440(5)$ \\
\hline Bond angle & $83.59(9)$ & $87.09(3)$ & $87.35(10)$ \\
\hline N1-Ni-S1 & $101.81(3)$ & $102.019(10)$ & $101.94(4)$ \\
\hline N1-Ni-N3 & $87.07(6)$ & are reported according & \\
\hline S2-Ni-S1 & to the atom labeling scheme in Figure 1, \\
\hline Metric parameters for & & \\
\hline which differs from prior & structural report. & & \\
\hline
\end{tabular}


Table S3. Bond distances and angles of complex 2.

\begin{tabular}{llll}
\hline Atoms & Distance $(\AA)$ & Atoms & Angle $\left(^{\circ}\right)$ \\
\hline Ni-N1 & $1.8528(8)$ & N1-Ni-N3 & $83.66(4)$ \\
Ni-N3 & $1.8580(8)$ & N1-Ni-S2 & $170.88(3)$ \\
Ni-S1 & $2.1617(3)$ & N3-Ni-S2 & $87.23(3)$ \\
Ni-S2 & N1-Ni-S1 & $87.09(3)$ \\
S1-C5 & $1.1576(2)$ & S2-Ni-S1 & $102.019(10)$ \\
N1-C1 & $1.7665(9)$ & C5-S1-Ni & $94.59(3)$ \\
N1-N2 & C1-N1-N2 & $119.80(8)$ \\
N2-C5 & $1.3694(11)$ & C1-N1-Ni & $115.46(6)$ \\
N5-C5 & $1.3222(12)$ & N2-N1-Ni & $124.64(6)$ \\
N5-C8 & $1.3456(12)$ & C5-N2-N1 & $110.47(8)$ \\
N5-C7 & $1.4453(13)$ & C5-N5-C8 & $124.17(9)$ \\
C1-C2 & $1.4570(13)$ & C5-N5-C7 & $118.67(8)$ \\
C1-C3 & $1.4658(13)$ & C8-N5-C7 & $117.15(8)$ \\
& $1.4857(13)$ & N1-C1-C2 & $112.75(8)$ \\
& & N1-C1-C3 & $123.71(9)$ \\
& & C2-C1-C3 & $123.52(8)$ \\
& & C1-C2-C4 & $122.36(8)$ \\
& & N2-C5-N5 & $116.74(8)$ \\
& & N2-C5-S1 & $123.18(7)$ \\
& & N5-C5-S1 & $120.06(7)$ \\
\hline
\end{tabular}


Table S4. Bond distances and angles of complex 3.

\begin{tabular}{|c|c|c|c|}
\hline Atoms & Distance $(\AA)$ & Atoms & Angle $\left({ }^{\circ}\right)$ \\
\hline Ni-N1 & $1.854(3)$ & N1-Ni-N3 & 83.65(13) \\
\hline Ni-N3 & $1.863(3)$ & N1-Ni-S2 & $170.63(10)$ \\
\hline $\mathrm{Ni}-\mathrm{S} 2$ & $2.1527(10)$ & N3-Ni-S2 & $87.12(9)$ \\
\hline Ni-S1 & $2.1542(11)$ & N1-Ni-S1 & $87.35(10)$ \\
\hline S1-C5 & $1.763(4)$ & S2-Ni-S1 & $101.94(4)$ \\
\hline N1-C1 & $1.310(5)$ & C5-S1-Ni & $94.17(13)$ \\
\hline N1-N2 & $1.381(4)$ & C1-N1-N2 & 120.5(3) \\
\hline N2-C5 & $1.308(5)$ & C1-N1-Ni & $115.3(2)$ \\
\hline N5-C5 & $1.350(5)$ & N2-N1-Ni & $124.2(2)$ \\
\hline N5-C7 & $1.440(5)$ & C5-N2-N1 & 109.8(3) \\
\hline F1-C8 & $1.338(5)$ & C5-N5-C7 & 123.5(3) \\
\hline F2-C8 & $1.339(4)$ & N1-C1-C2 & 112.6(3) \\
\hline F3-C8 & $1.347(4)$ & N1-C1-C3 & 124.1(3) \\
\hline C1-C2 & $1.474(5)$ & C2-C1-C3 & 123.3(3) \\
\hline \multirow[t]{11}{*}{ C1-C3 } & $1.487(5)$ & C1-C2-C4 & 122.6(3) \\
\hline & & N2-C5-N5 & 119.3(3) \\
\hline & & N2-C5-S1 & 124.4(3) \\
\hline & & N5-C5-S1 & $116.2(3)$ \\
\hline & & N5-C7-C8 & 111.7(3) \\
\hline & & F1-C8-F2 & 106.7(3) \\
\hline & & F1-C8-F3 & 106.7(3) \\
\hline & & F2-C8-F3 & $106.0(3)$ \\
\hline & & F1-C8-C7 & 112.6(3) \\
\hline & & F2-C8-C7 & 111.4(3) \\
\hline & & F3-C8-C7 & 112.8(3) \\
\hline
\end{tabular}




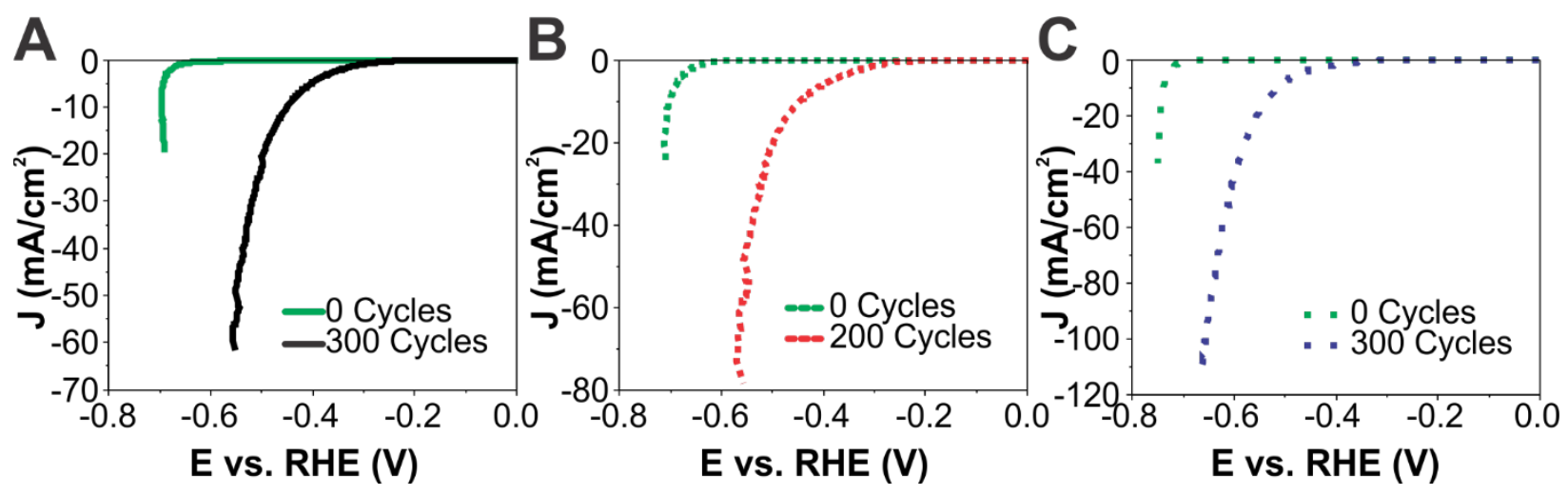

Figure S6. Effects of reductive cycling from 0 to $-0.8 \mathrm{~V}$ vs. RHE at $50 \mathrm{mV} / \mathrm{s}$ on the performance of the three modified electrodes. Vertical scale is expanded to show peak cathodic current for each electrode.

(A) Polarization curves for GC-1 which exhibits peak HER performance after 300 cycles. (B) Polarization curves for GC-2 which exhibits peak HER performance after 200 cycles. (C) Polarization curves for GC-3 which exhibits peak HER performance after 300 cycles. 

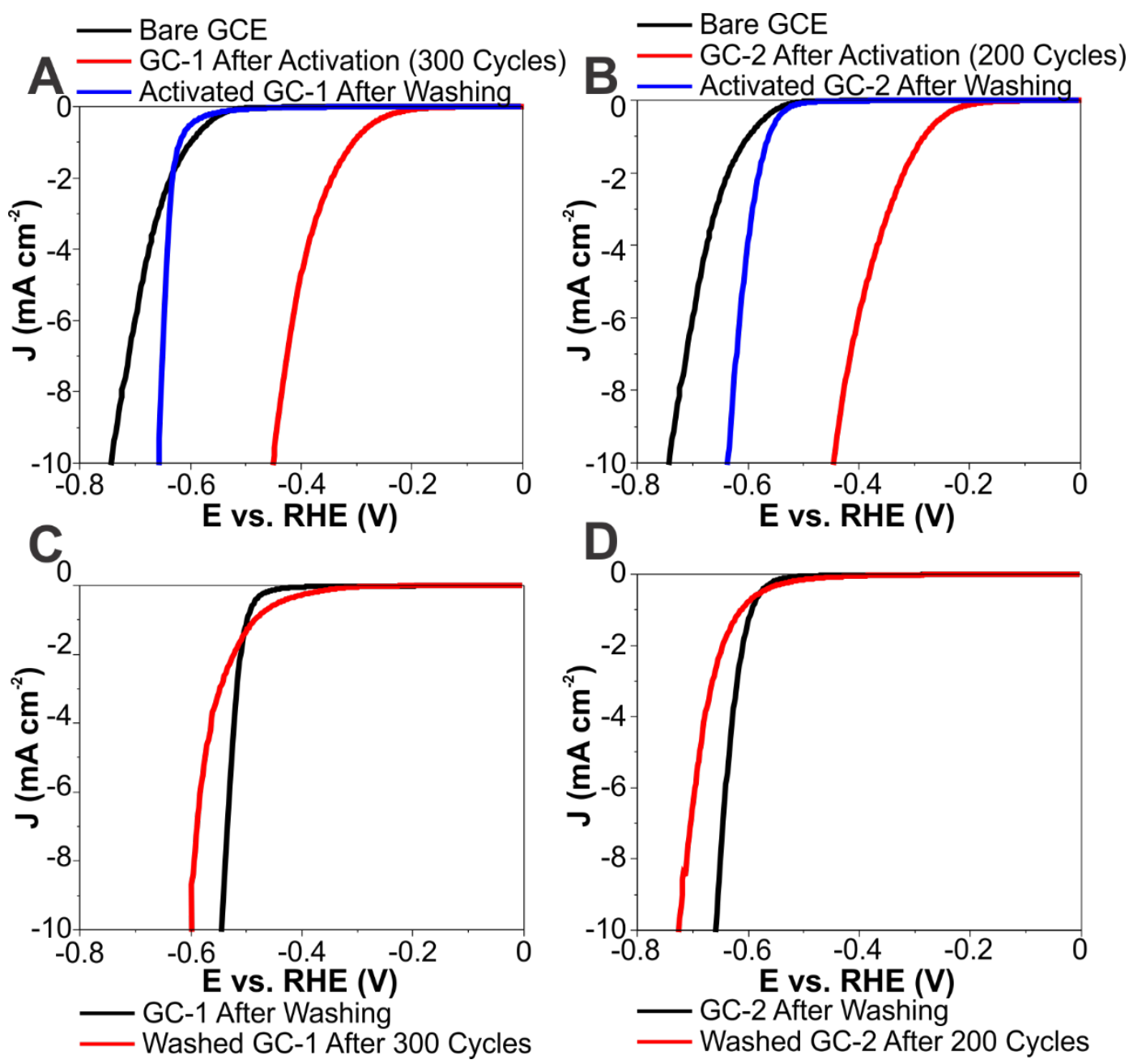

Figure S7. Effect on HER performance of dipping modified electrodes in acetonitrile for $\mathbf{3 0}$ seconds at $800 \mathrm{RPM}$ rotation.

(A) GC-1 conditioned to peak activity (300 cycles, red trace) and then washed in acetonitrile (blue trace) and compared to bare glassy carbon electrode (black trace). (B) GC-2 conditioned to peak activity (200 cycles, red trace) and then washed in acetonitrile (blue trace) and compared to glassy carbon (black trace). (C) GC-1 washed in acetonitrile before any conditioning (black trace) and subsequently conditioned to 300 cycles (red trace). (D) GC-2 washed in acetonitrile before any conditioning (black trace) and subsequently conditioned to 200 cycles (red trace). 

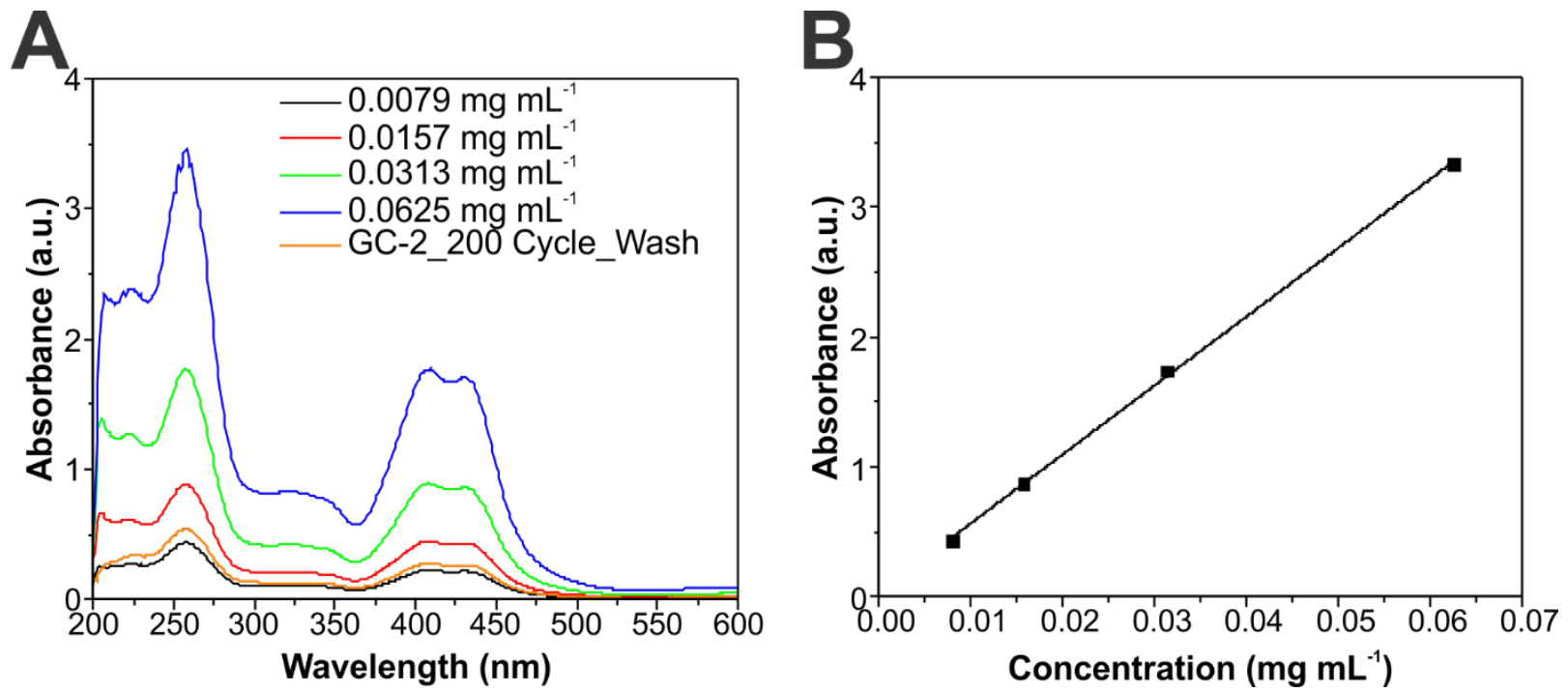

Figure S8. UV-Visible Spectroscopy data for complex 2 in solution

(A) UV-Vis spectra for complex 2 in acetonitrile solutions of known concentration and of unknown concentration after being washed off GC-2 after conditioning to peak activity (200 cycles). (B) Calibration curve constructed from measured absorbances and known concentrations of standard acetonitrile solutions of 2.

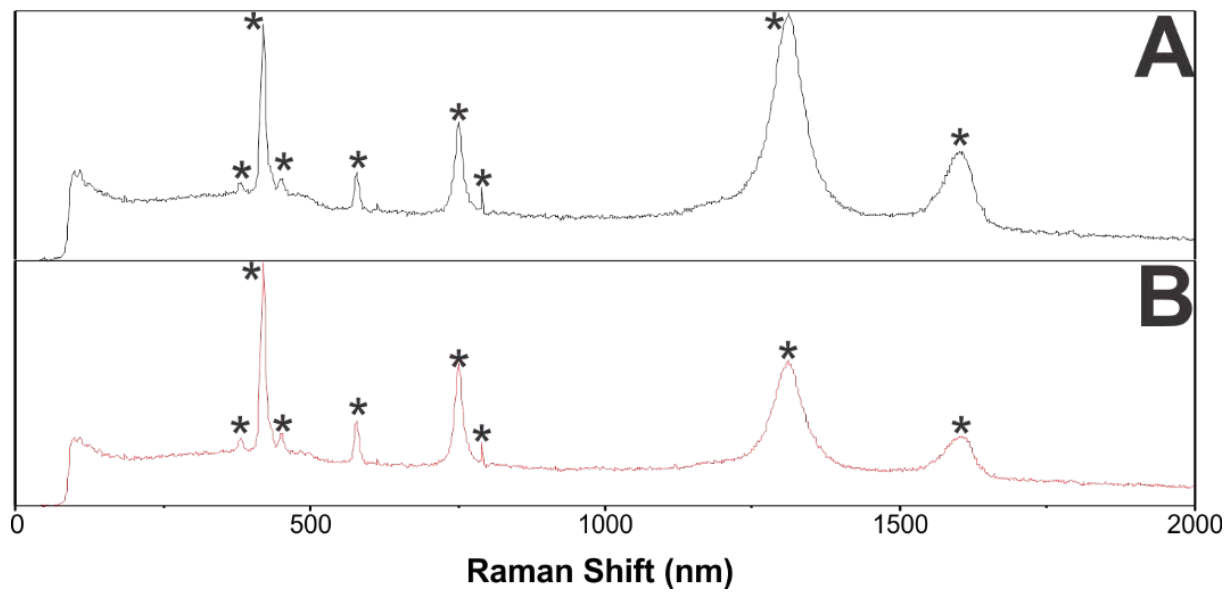

Figure S9. Raman spectra of various electrodes and organic solutions with bands labelled.

Raman spectrum of (A) Glassy carbon electrode with Nafion film. (B) Bare glassy carbon electrode. Peaks labelled with * are attributed to the glassy carbon electrode. 


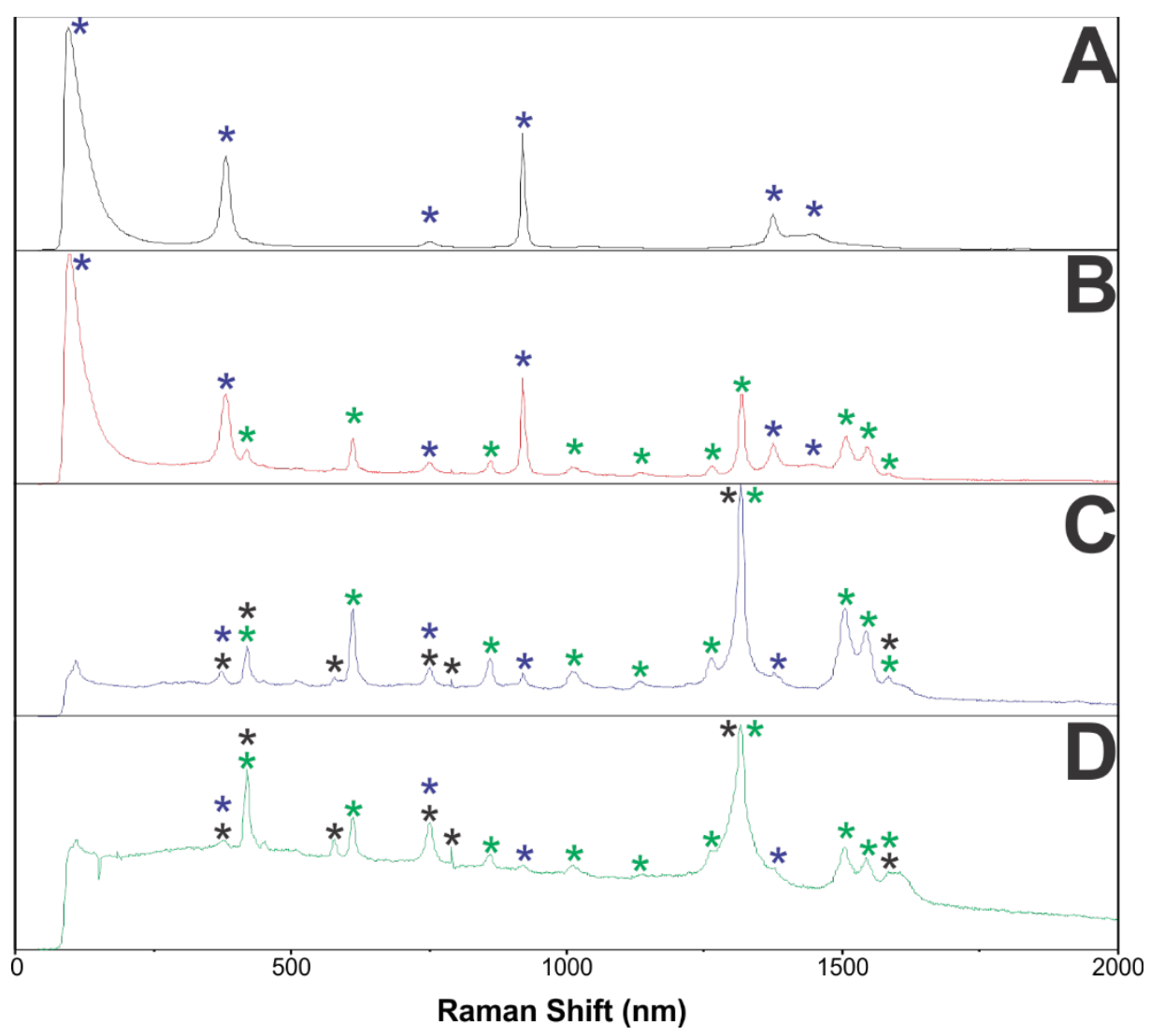

Figure S10. Raman spectra of various electrodes and organic solutions with bands labelled.

Raman spectrum of (A) Acetonitrile. (B) Complex 2 in acetonitrile ink with Nafion. (C) GC-2 as deposited. (D) GC-2 after conditioning to peak activity (200 cycles). Peaks labelled with * are attributed to the glassy carbon electrode. Peaks labelled with * are attributed to acetonitrile. Peaks labelled with * are attributed to complex 2. 


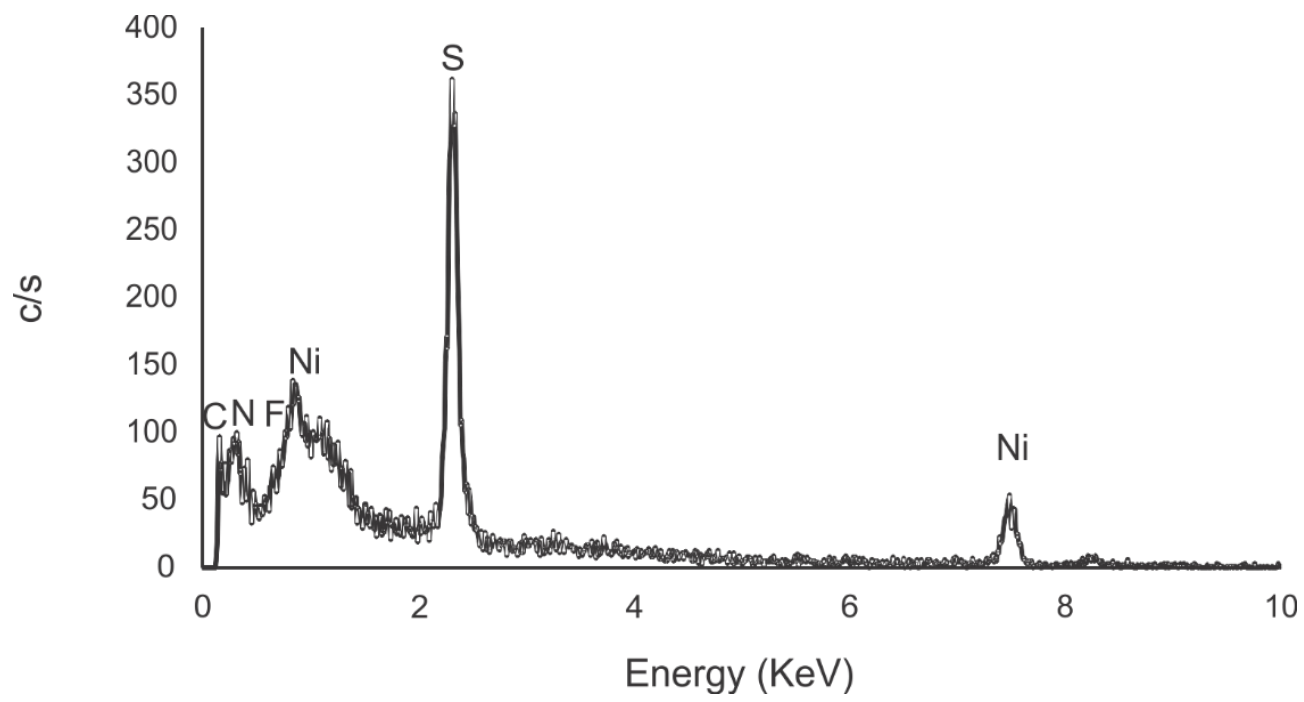

Figure S11. EDS of GC-2 before reductive cycling.

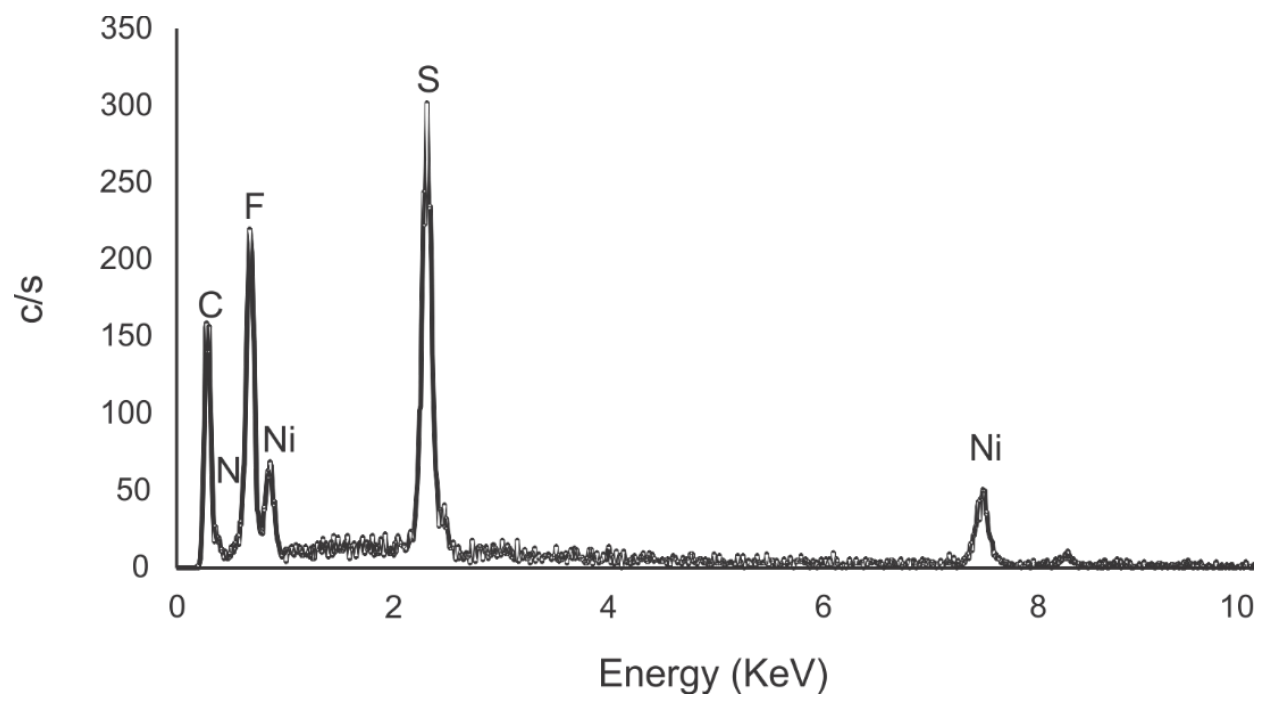

Figure S12. EDS of GC-2 after 1000 cycles. 

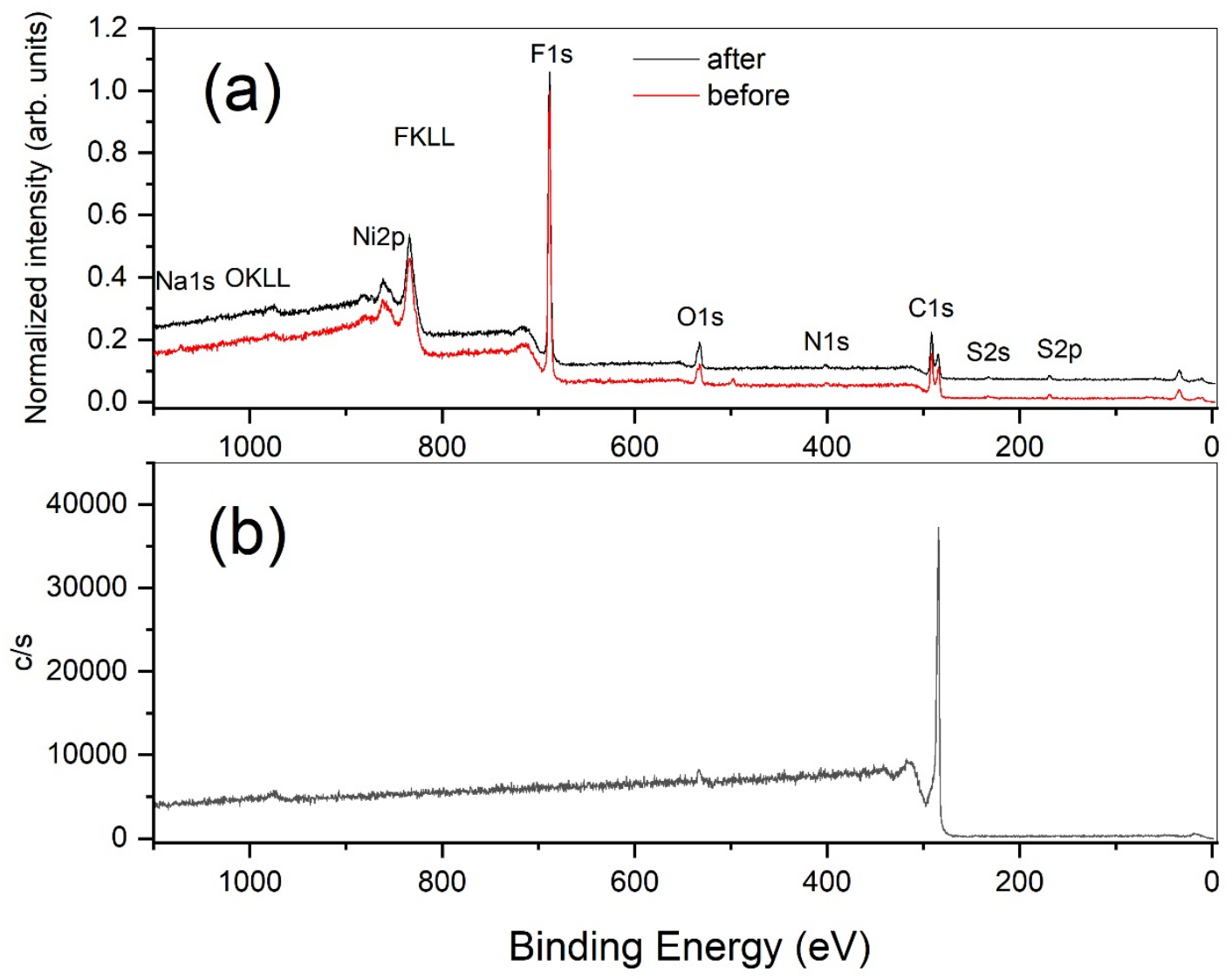

Figure S13. Survey scans of the GC-2 electrode before (red) and after (black) conditioning to 1000 cycles (a) and of a pristine GCE (b).

Table S5. Atomic \% of Ni2p, S2p and Ni1s before and after conditioning (1000 cycles).

\begin{tabular}{|l|l|l|l|}
\cline { 2 - 4 } \multicolumn{1}{l|}{} & N1s, at\% & S2p, at\% & Ni2p, at\% \\
\hline Before & 61.3 & 26.74 & 11.96 \\
\hline After & 66.74 & 23.35 & 9.91 \\
\hline
\end{tabular}


Table S6. XPS fitting parameters for nickel (Figure 8A).

\begin{tabular}{|c|c|c|c|c|c|c|c|c|c|}
\hline Band & Pos & PosSep & B_FWHM & FWHM & Height & \%Gauss & Area & \%Area & ChiSquared \\
\hline 1 & 853.38 & 0 & 3.12 & 3.12 & 1459 & 80 & 5309 & 37.12 & 1.94 \\
\hline 2 & 859.24 & 5.86 & 6.72 & 6.72 & 482 & 80 & 3775 & 26.4 & \\
\hline 3 & 870.81 & 17.43 & 3.37 & 3.37 & 675 & 80 & 2654 & 18.56 & \\
\hline 4 & 876.33 & 22.95 & 6.39 & 6.39 & 344 & 80 & 2564 & 17.93 & \\
\hline
\end{tabular}

Table S7. XPS fitting parameters for sulfur (Figure 8B).

\begin{tabular}{|c|c|c|c|c|c|c|c|c|c|}
\hline Band & Pos & PosSep & B_FWHM & FWHM & Height & \%Gauss & Area & \%Area & ChiSquared \\
\hline 1 & 162.93 & 0 & 2.6 & 2.6 & 211 & 80 & 640 & 56.12 & 1.35 \\
\hline 2 & 164.13 & 1.2 & 2.6 & 2.6 & 109 & 87 & 320 & 28.06 & \\
\hline 3 & 168.33 & 5.4 & 2.7 & 2.7 & 37 & 70 & 120 & 10.54 & \\
\hline 4 & 169.53 & 6.6 & 2.65 & 2.65 & 19 & 70 & 60 & 5.27 & \\
\hline
\end{tabular}

Table S8. XPS fitting parameters for nickel (Figure 10A).

\begin{tabular}{|c|c|c|c|c|c|c|c|c|c|}
\hline Band & Pos & PosSep & B_FWHM & FWHM & Height & \%Gauss & Area & \%Area & ChiSquared \\
\hline 1 & 853.31 & 0 & 2.84 & 2.84 & 6436 & 90 & 20406 & 29.53 & 2.81 \\
\hline 2 & 858.1 & 4.79 & 8.79 & 8.79 & 2405 & 90 & 23572 & 34.11 & \\
\hline 3 & 870.58 & 17.28 & 3.96 & 3.96 & 2656 & 90 & 11718 & 16.95 & \\
\hline 4 & 876.2 & 22.89 & 8.94 & 8.94 & 1346 & 90 & 13417 & 19.41 & \\
\hline
\end{tabular}

Table S9. XPS fitting parameters for sulfur (Figure 10B).

\begin{tabular}{|c|c|c|c|c|c|c|c|c|c|}
\hline Band & Pos & PosSep & B_FWHM & FWHM & Height & \%Gauss & Area & \%Area & ChiSquared \\
\hline 1 & 162.32 & 0 & 2.57 & 2.57 & 1571 & 100 & 4293 & 51.15 & 1.71 \\
\hline 2 & 163.52 & 1.2 & 2.57 & 2.57 & 718 & 80 & 2146 & 25.57 & \\
\hline 3 & 164.48 & 2.16 & 3.2 & 3.2 & 221 & 60 & 895 & 10.66 & \\
\hline 4 & 165.68 & 3.36 & 3.2 & 3.2 & 116 & 71 & 447 & 5.33 & \\
\hline 5 & 168.22 & 5.9 & 2.7 & 2.7 & 124 & 70 & 408 & 4.86 & \\
\hline 6 & 169.42 & 7.1 & 2.7 & 2.7 & 65 & 79 & 204 & 2.43 & \\
\hline
\end{tabular}



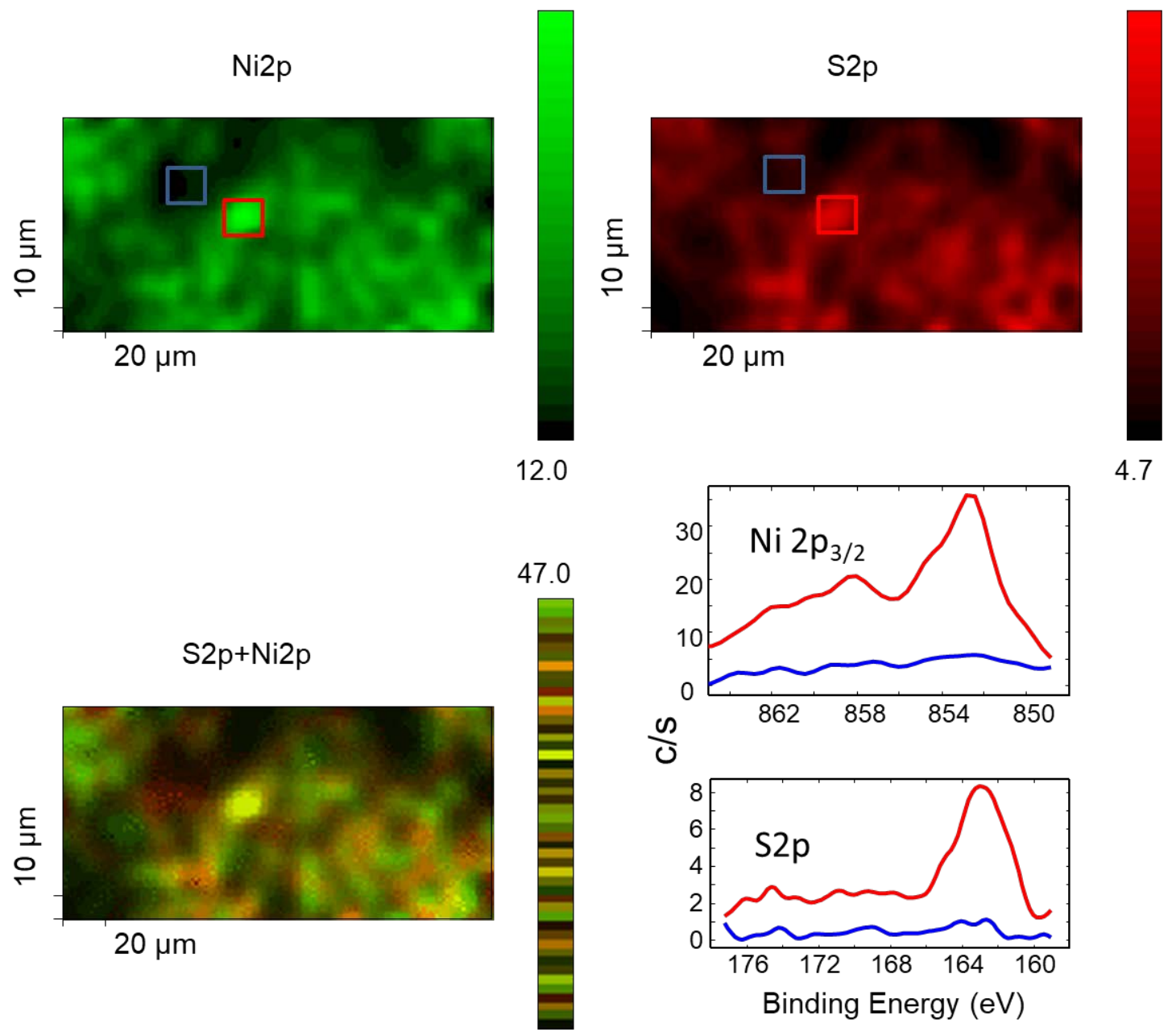

4.7
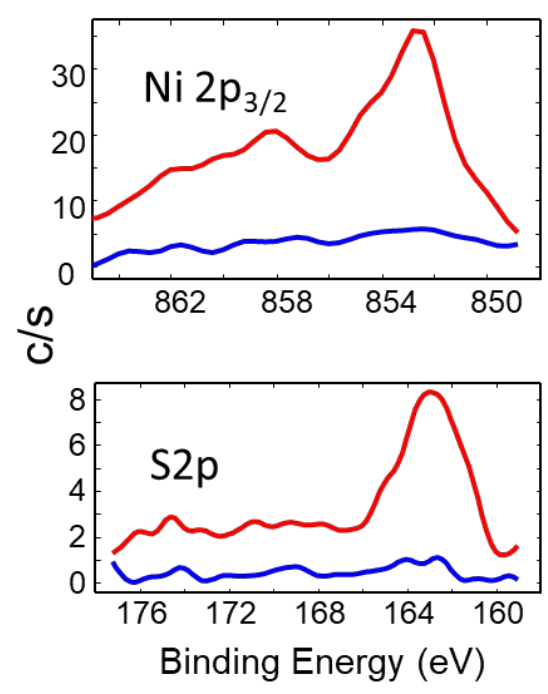

0.0

Figure S14. Elemental mapping of nickel and sulfur.

Elemental mapping of nickel (top left), sulfur (top right), and an overlay of both (bottom left) at $200 \times 100 \mu \mathrm{m}$ scanned area and XPS spectra (right bottom) in which the red trace and blue trace correspond to the pixels integrated through red and blue squares respectively.

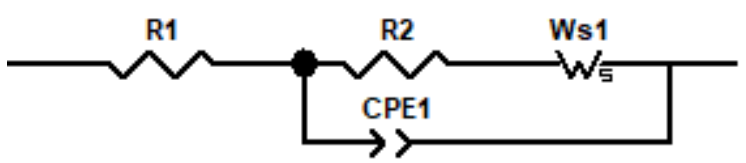

Figure S15. Equivalent circuit model used to fit EIS data. 
Table S10. Parameters obtained by fitting frequency response analysis (FRA) data to simulated RCW circuit.

Zview software ${ }^{2}$ was used to fit data. $Q$ is the magnitude of the CPE, $R_{c}$ is the charge transfer resistance, $R_{S}$ is approximate solution resistance, $n$ is the CPE exponent, and $C$ is the capacitance. Capacitance values were calculated using $C=R_{c} \frac{1-n}{n} * Q^{\frac{1}{n}} \cdot 3-7$

\begin{tabular}{l|lll|lll|lll}
$\mathbf{G C}$ & \multicolumn{3}{|c}{$\mathbf{1}$} & \multicolumn{3}{|c}{$\mathbf{2}$} & \multicolumn{3}{|c}{$\mathbf{3}$} \\
\# Cycles & $\mathbf{0}$ & $\mathbf{3 0 0}$ & $\mathbf{1 0 0 0}$ & $\mathbf{0}$ & $\mathbf{2 0 0}$ & $\mathbf{1 0 0 0}$ & $\mathbf{0}$ & $\mathbf{3 0 0}$ & $\mathbf{1 0 0 0}$ \\
$\mathbf{Q}\left(\mathbf{F ~ s}^{\mathbf{n}-\mathbf{1}}\right)$ & $2.43 \mathrm{E}-6$ & $4.52 \mathrm{E}-6$ & $4.46 \mathrm{E}-6$ & $2.63 \mathrm{E}-6$ & $6.10 \mathrm{E}-6$ & $6.33 \mathrm{E}-6$ & $3.55 \mathrm{E}-5$ & $3.20 \mathrm{E}-5$ & $5.14 \mathrm{E}-5$ \\
$\mathbf{R}_{\mathbf{C}}(\mathbf{\Omega})$ & 25099 & 270 & 374 & 18843 & 158 & 374 & 54764 & 631 & 1093 \\
$\mathbf{R}_{\mathbf{s}}(\boldsymbol{\Omega})$ & 26.22 & 20.12 & 17.99 & 16.80 & 15.09 & 13.9 & 11.14 & 6.44 & 5.78 \\
$\mathbf{N}$ & 0.908 & 0.929 & 0.913 & 0.916 & 0.893 & 0.864 & 0.951 & 0.729 & 0.717 \\
$\mathbf{C ~ ( F ) ~}$ & $1.83 \mathrm{E}-6$ & $2.71 \mathrm{E}-6$ & $2.43 \mathrm{E}-6$ & $2.00 \mathrm{E}-6$ & $2.65 \mathrm{E}-6$ & $2.49 \mathrm{E}-6$ & $3.67 \mathrm{E}-5$ & $7.52 \mathrm{E}-6$ & $1.66 \mathrm{E}-5$ \\
$\chi^{2}$ & 0.00015 & 0.00121 & 0.00141 & 0.00072 & 0.00057 & 0.00057 & 0.03272 & 0.00163 & 0.00434
\end{tabular}
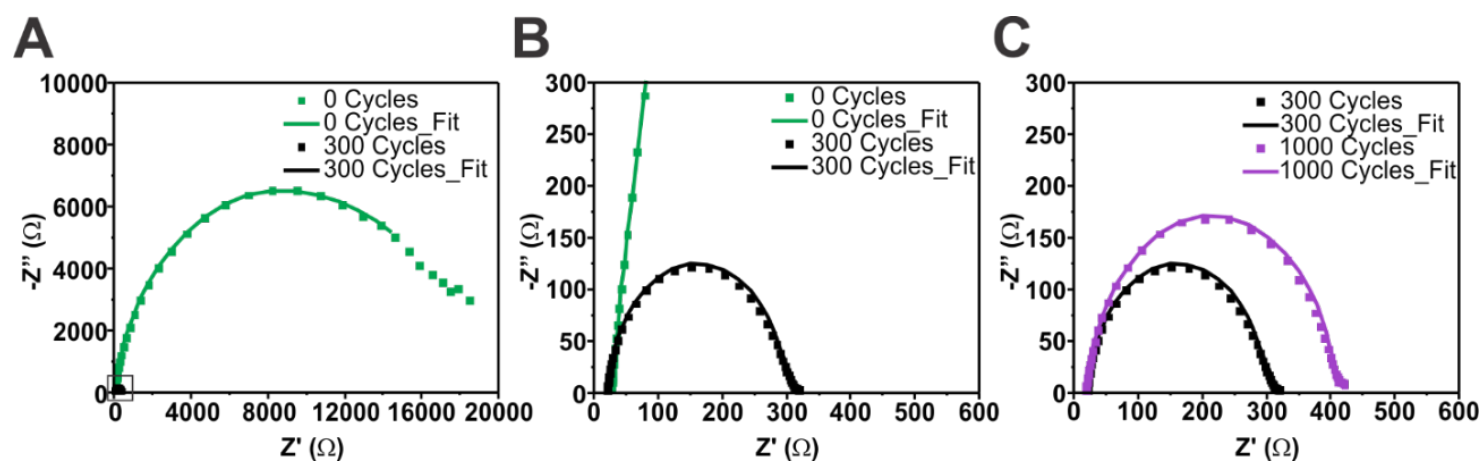

Figure S16. Nyquist plots showing the effects of reductive cycling from 0 to $-\mathbf{0 . 8} \mathrm{V}$ vs. RHE at $50 \mathrm{mV} / \mathrm{sec}$ on the impedance of GC-1.

Solid traces represent fitted impedance data, while dotted traces represent actual data. (A) Nyquist plot showing impedance at 0 cycles (before any reductive cycling) and after 300 cycles (peak activity, in small frame). (B) The same data as in (A), but with axes scaled down to show impedance after 300 cycles in greater detail. (C) Comparison of Nyquist plots for GC-1 after 300 cycles and 1000 cycles. 

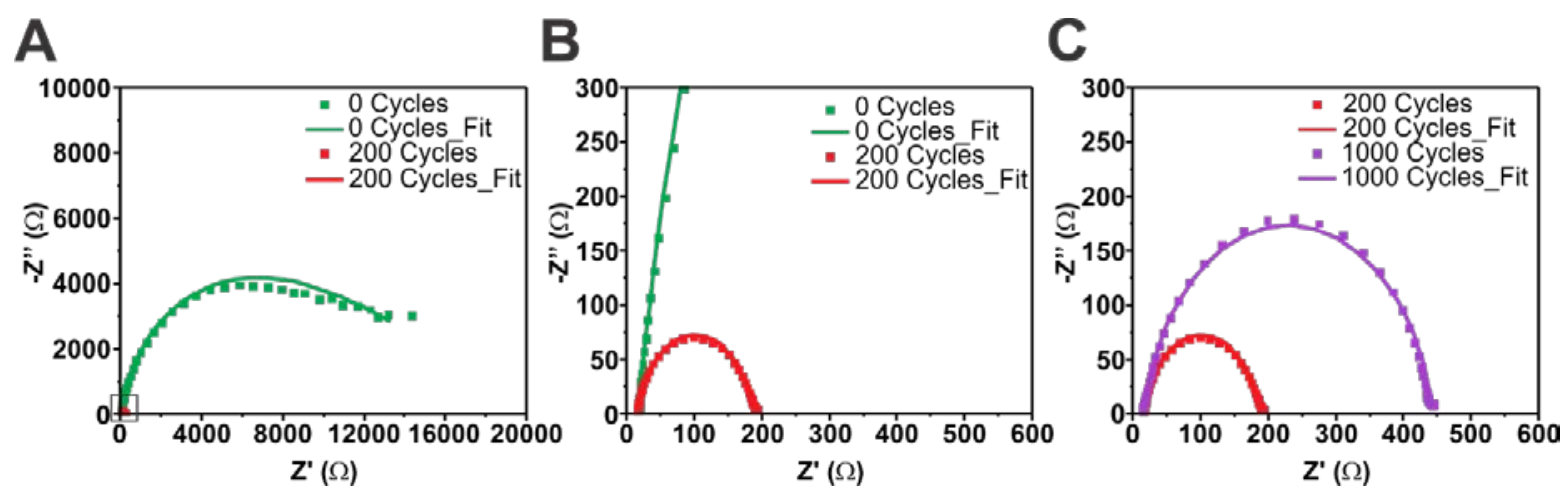

Figure S17. Nyquist plots showing the effects of reductive cycling from 0 to $-0.8 \mathrm{~V}$ vs. RHE at $50 \mathrm{mV} / \mathrm{sec}$ on the impedance of GC-2.

Solid traces represent fitted impedance data, while dotted traces represent actual data. (A) Nyquist plot showing impedance at 0 cycles (before any reductive cycling) and after 200 cycles (peak activity, in small frame). (B) The same data as in (A), but with axes scaled down to show impedance after 200 cycles in greater detail. (C) Comparison of Nyquist plots for GC-2 after 200 and 1000 cycles.
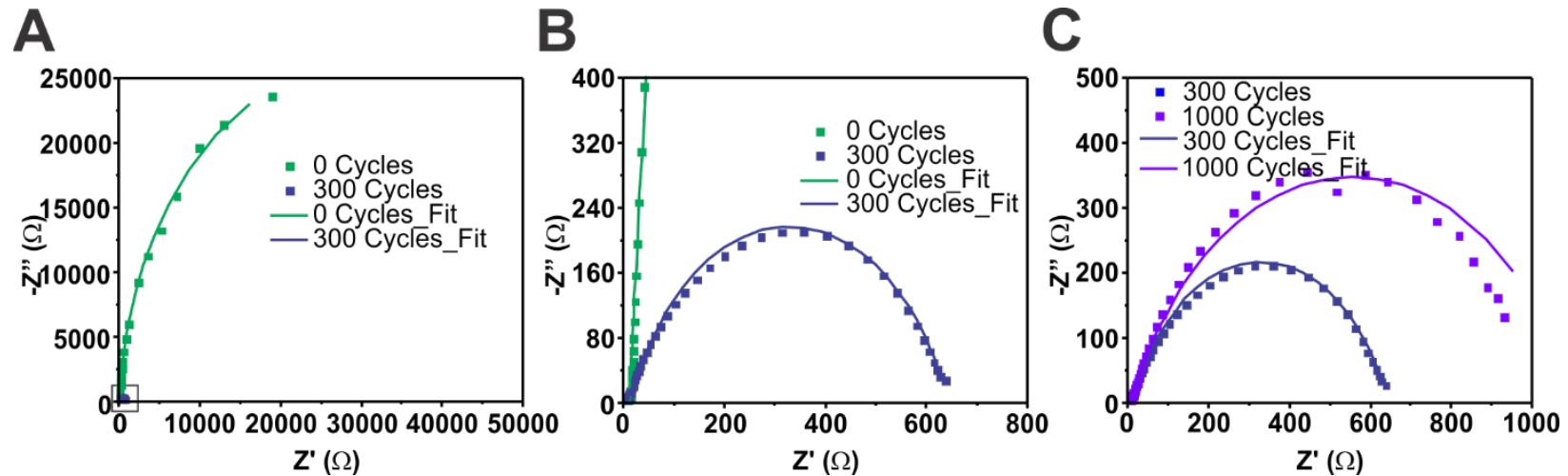

Figure S18. Nyquist plots showing the effects of cycling from 0 to $-0.8 \mathrm{~V}$ vs. RHE at $50 \mathrm{mV} / \mathrm{sec}$ on the impedance of GC-3.

Solid traces represent fitted impedance data, while dotted traces represent actual data. (A) Nyquist plot showing impedance at 0 cycles (before any reductive cycling) and after 300 cycles (peak activity, in small frame). (B) The same data as in (A), but with axes scaled down to show impedance after 300 cycles in greater detail. (C) Comparison of Nyquist plots for GC-3 after 300 and 1000 cycles. 

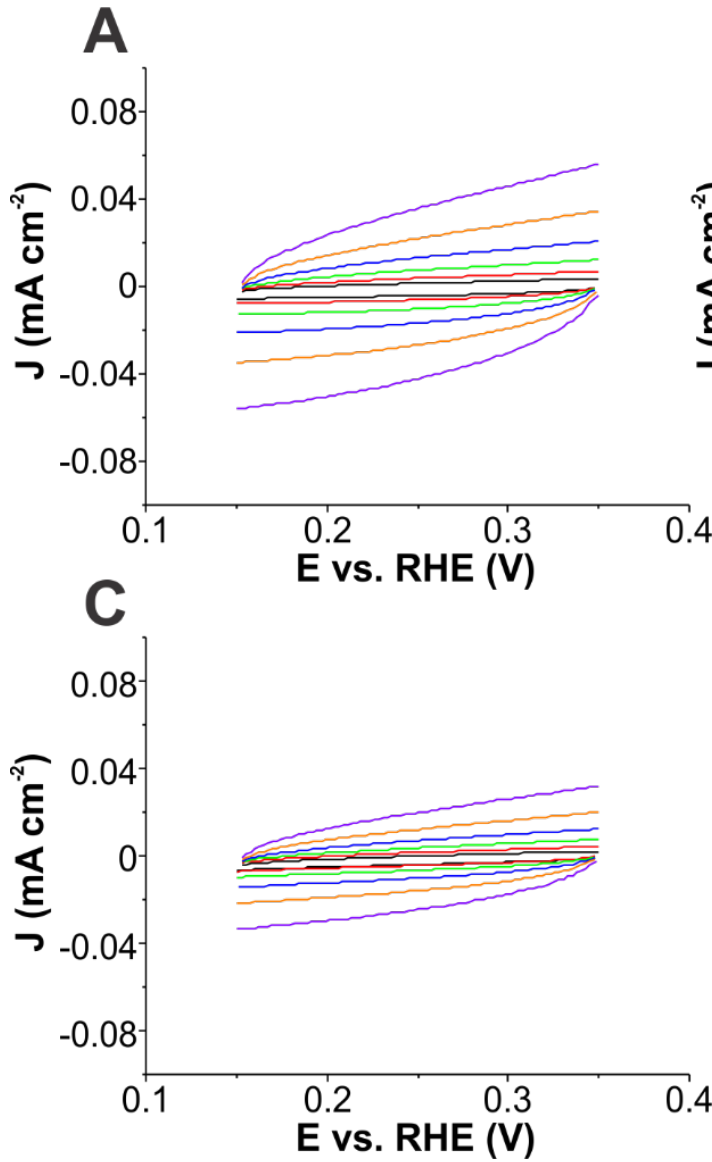

B

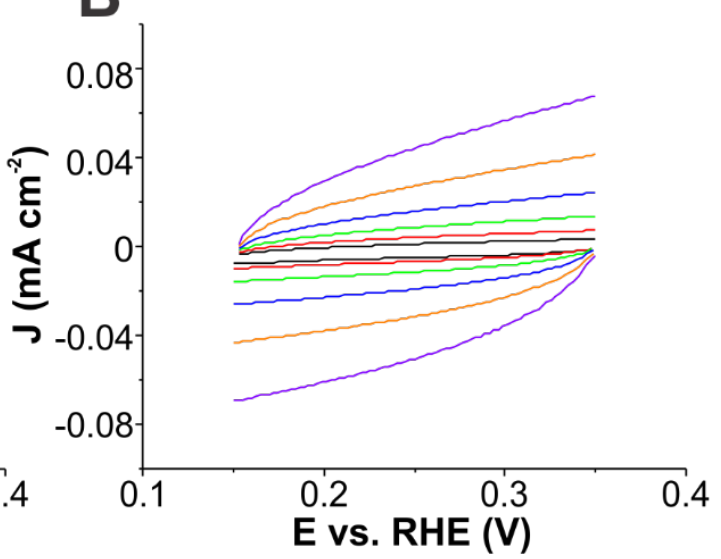

Figure S19. Cyclic voltammetry plots showing the capacitive effects of varying scan speed over a non-Faradaic potential range for GC $1-3$ as-deposited.

(A) GC-1 at 0 cycles. (B) GC-2 at 0 cycles. (C) GC-3 at 0 cycles 


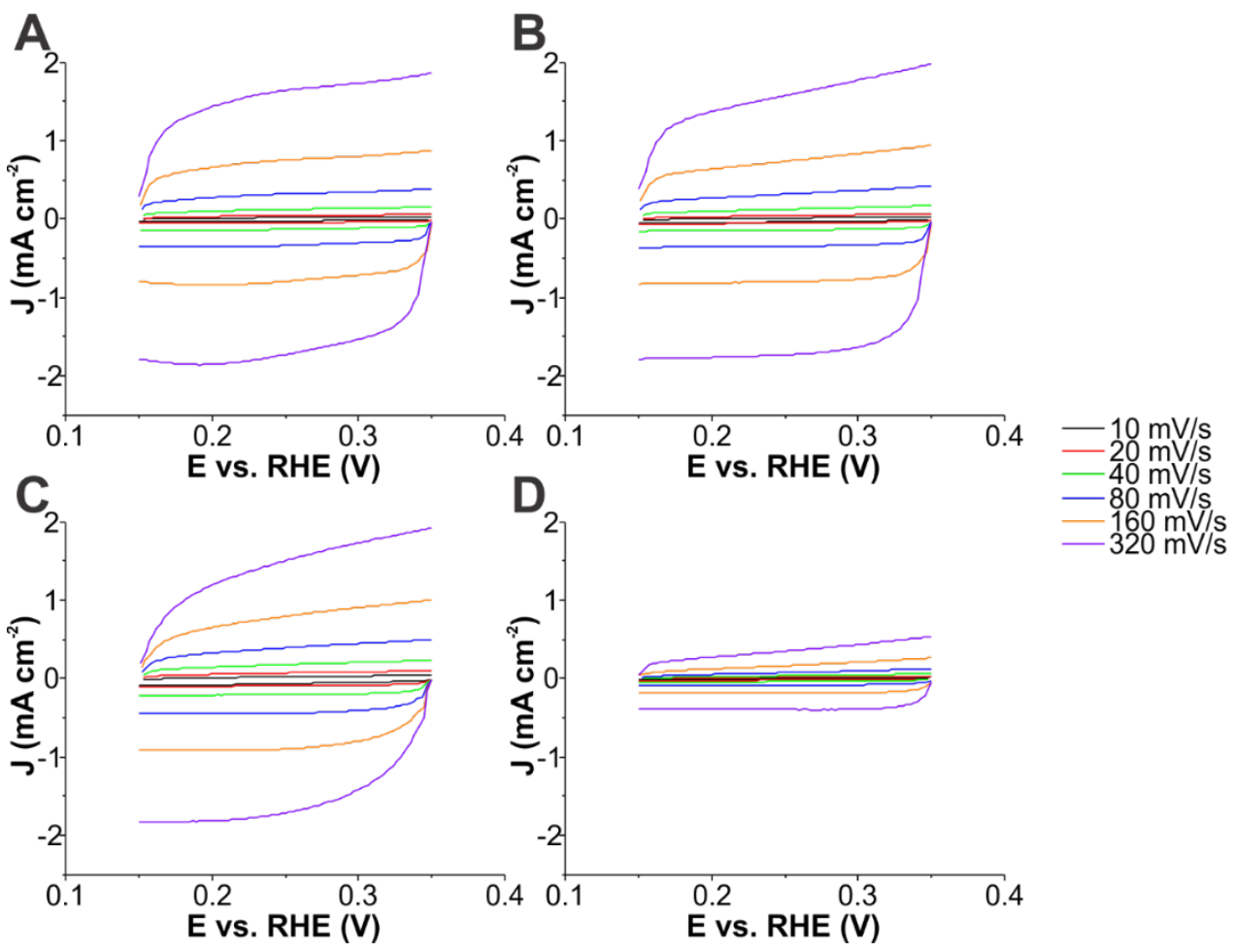

Figure S20. Cyclic voltammetry plots showing the capacitive effects of varying scan speed over a non-Faradaic potential range for GC $1-3$ after cycling to peak activity.

(A) GC-1 after 300 cycles. (B) GC-2 after 200 cycles. (C) GC-3 after 300 cycles. (D) Bare glassy carbon electrode.

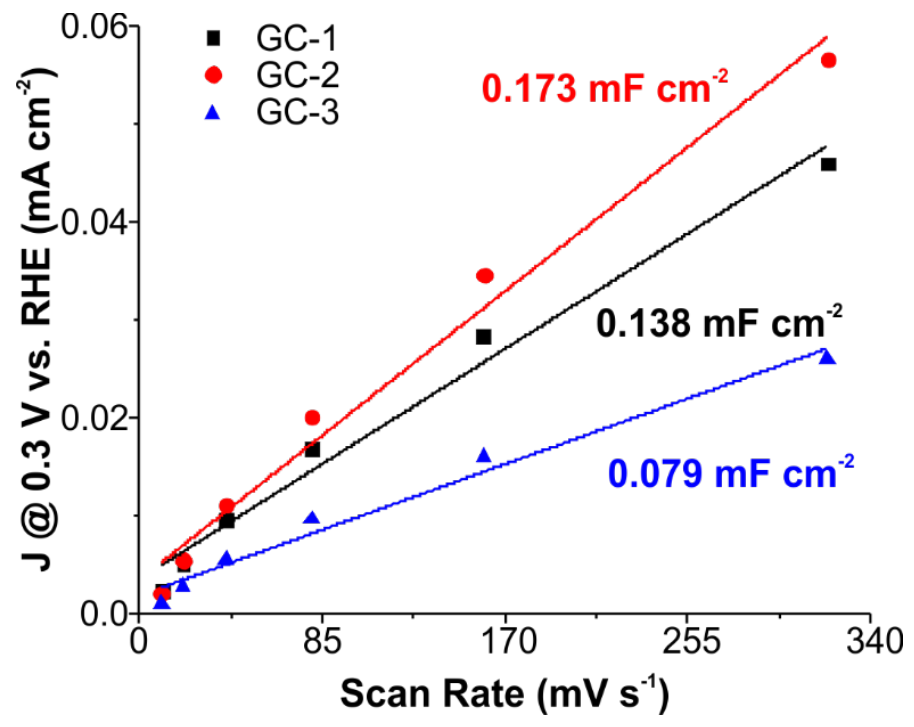

Figure S21. Plot of average current density at $0.3 \mathrm{~V}$ vs. RHE as measured during anodic sweep as a function of scan rate for as-deposited electrodes.

Slopes of the traces give the approximate capacitances of each modified electrode per unit geometric area (denoted in the same color as the corresponding trace). 


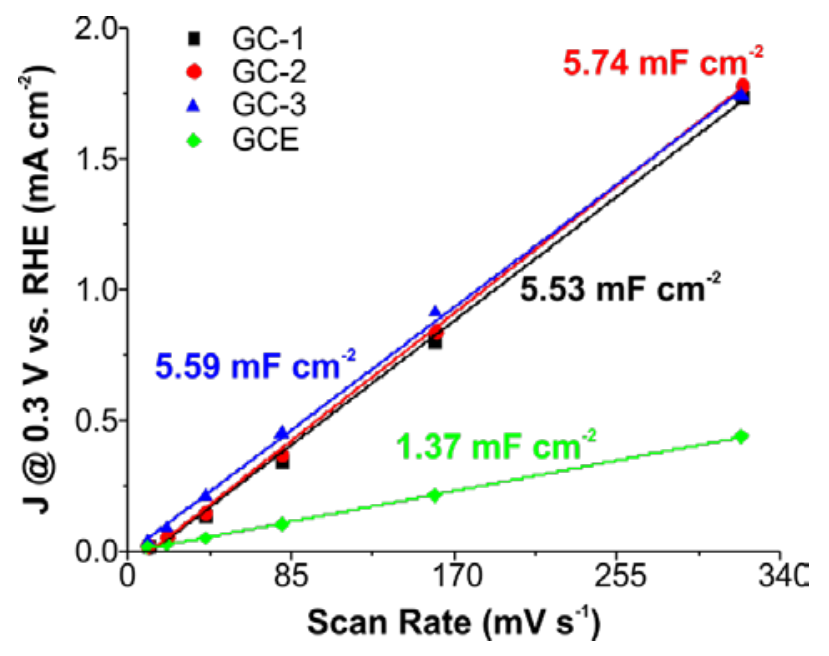

Figure S22. Plot of average current density at $0.3 \mathrm{~V}$ vs. RHE as measured during anodic sweep as a function of scan rate for GC 1 - 3 after cycling to peak activity.

Slopes of the traces give the approximate capacitances of each modified electrode per unit geometric area. The black trace corresponds to GC-1 after 300 cycles, the red trace corresponds to GC-2 after 200 cycles, and the blue trace corresponds to GC-3 after 300 cycles. 

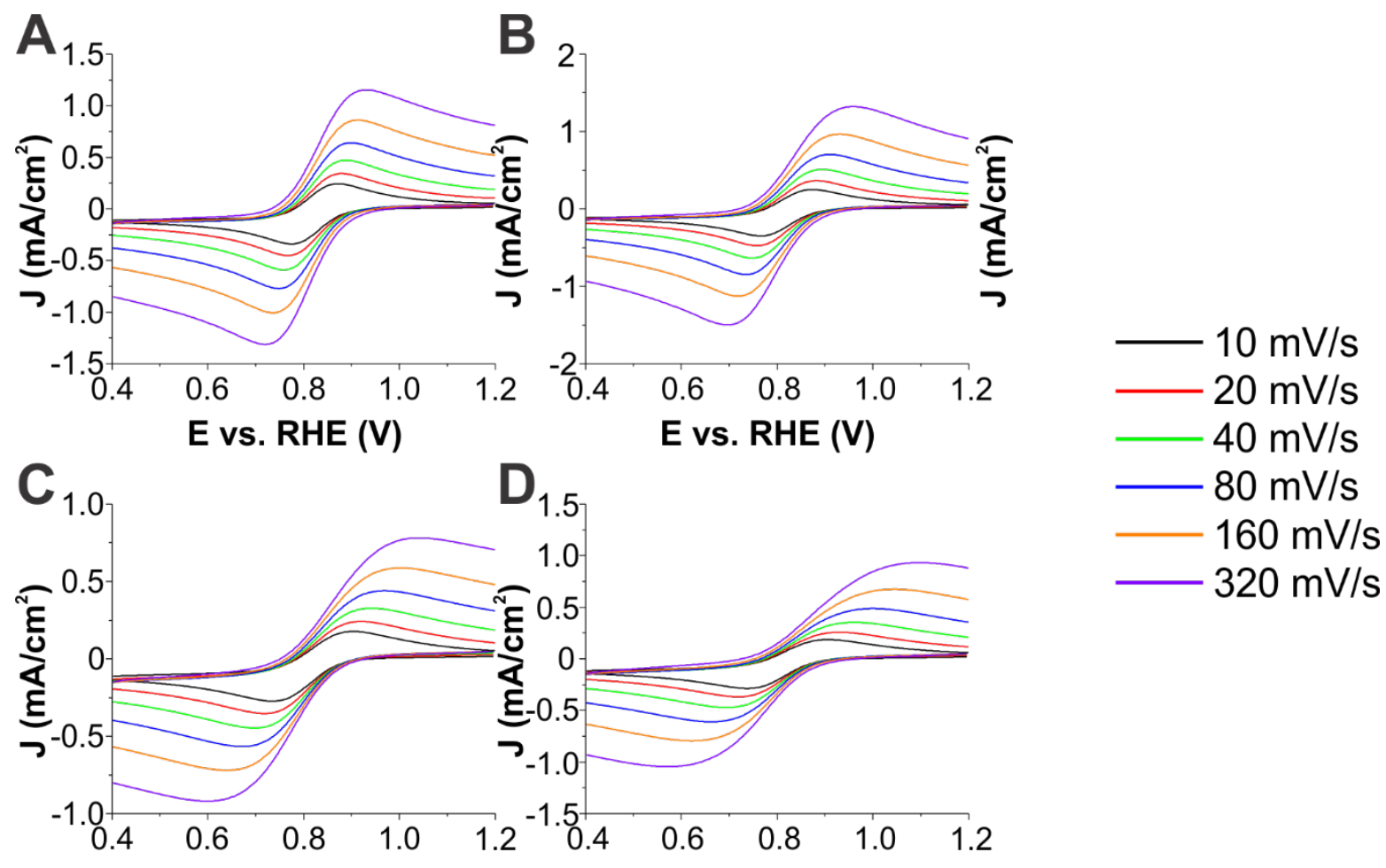

E vs. RHE (V)

$\mathbf{D}_{1.5}$

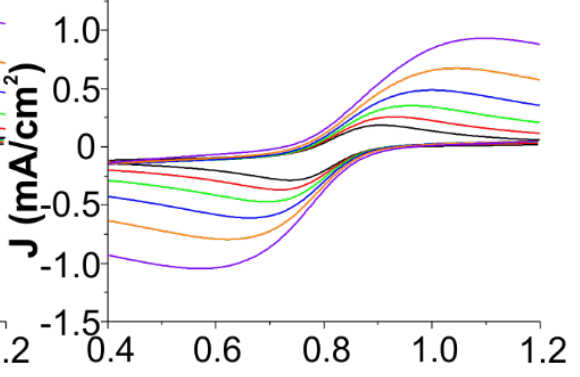

E vs. RHE (V)

Figure S23. Oxidation (top peak) and reduction (bottom peak) of ferricyanide redox couple at $5 \mathrm{mM}$ in $0.1 \mathrm{M}$ potassium nitrate solution.

(A) GC-1 as-deposited. (B) GC-1 after conditioning to 300 cycles. (C) GC-2 as-deposited. (D) GC-2 after conditioning to 200 cycles. 


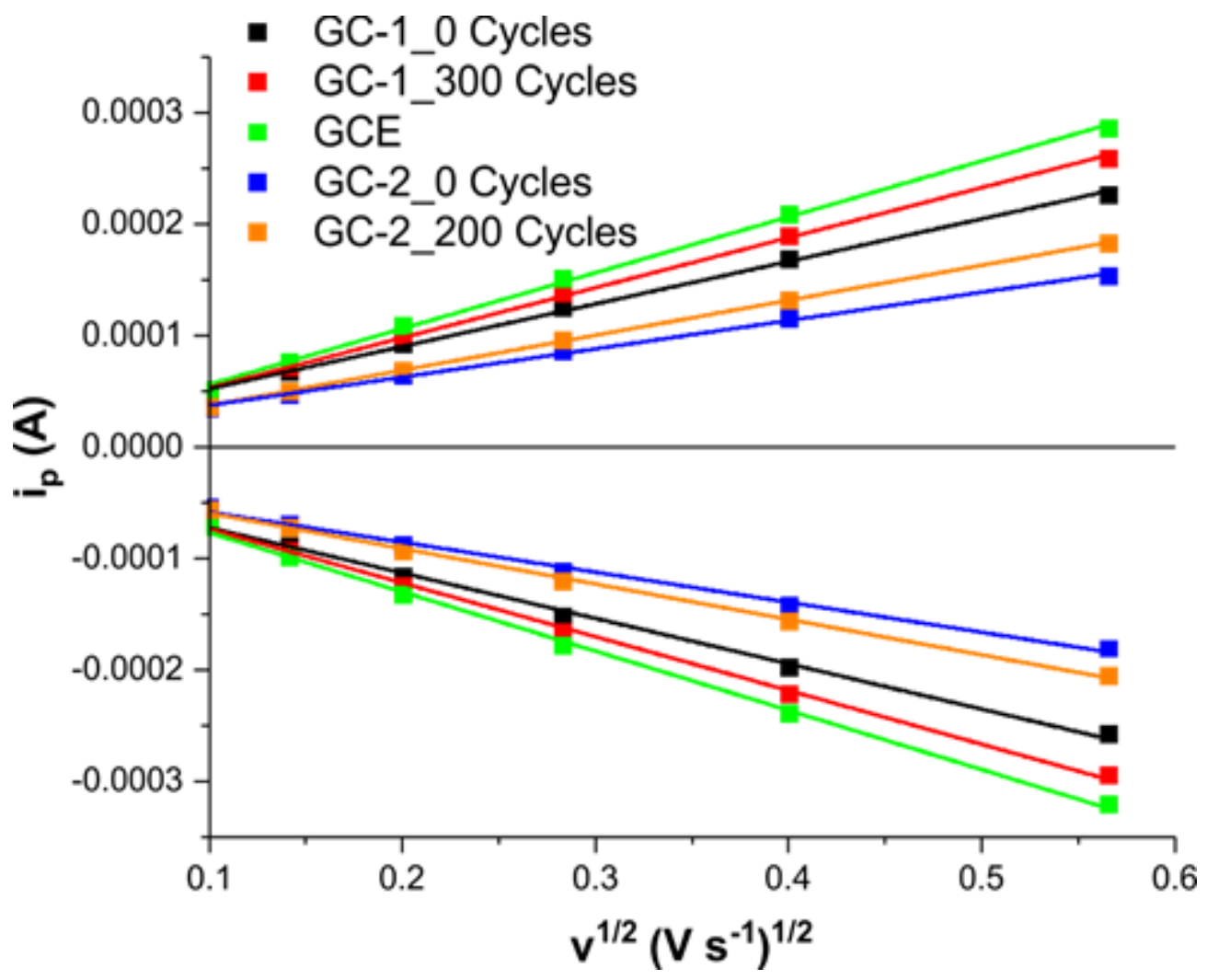

Figure S24. Plot of maximum current (top, for anodic sweeps) and minimum current (bottom, for cathodic sweeps) associated with ferricyanide reduction/oxidation cyclic voltammetry experiment as a function of the square root of scan rate.

Table S11. Slopes of the traces of maximum/minimum current densities as a function of the square root of scan rate for ferricyanide oxidation/reduction experiments as well as areas calculated from the Randles-Sevcik equation.

\begin{tabular}{|l|l|l|l|l|}
\hline & \multicolumn{2}{|l|}{ Anodic } & \multicolumn{2}{l|}{ Cathodic } \\
\hline Material & Slope & Area $\left(\mathbf{c m}^{\mathbf{2}}\right)$ & Slope & Area $\left.\mathbf{( c m}^{\mathbf{2}}\right)$ \\
\hline GC-1_300 Cycles & $4.5 \times 10^{-4}$ & 0.12 & $-4.8 \times 10^{-4}$ & 0.14 \\
\hline GC-1_0 Cycles & $3.8 \times 10^{-4}$ & 0.11 & $-4.1 \times 10^{-4}$ & 0.12 \\
\hline GC-2_200 Cycles & $3.1 \times 10^{-4}$ & 0.09 & $-3.2 \times 10^{-4}$ & 0.09 \\
\hline GC-2_0 Cycles & $2.5 \times 10^{-4}$ & 0.07 & $-2.7 \times 10^{-4}$ & 0.08 \\
\hline
\end{tabular}




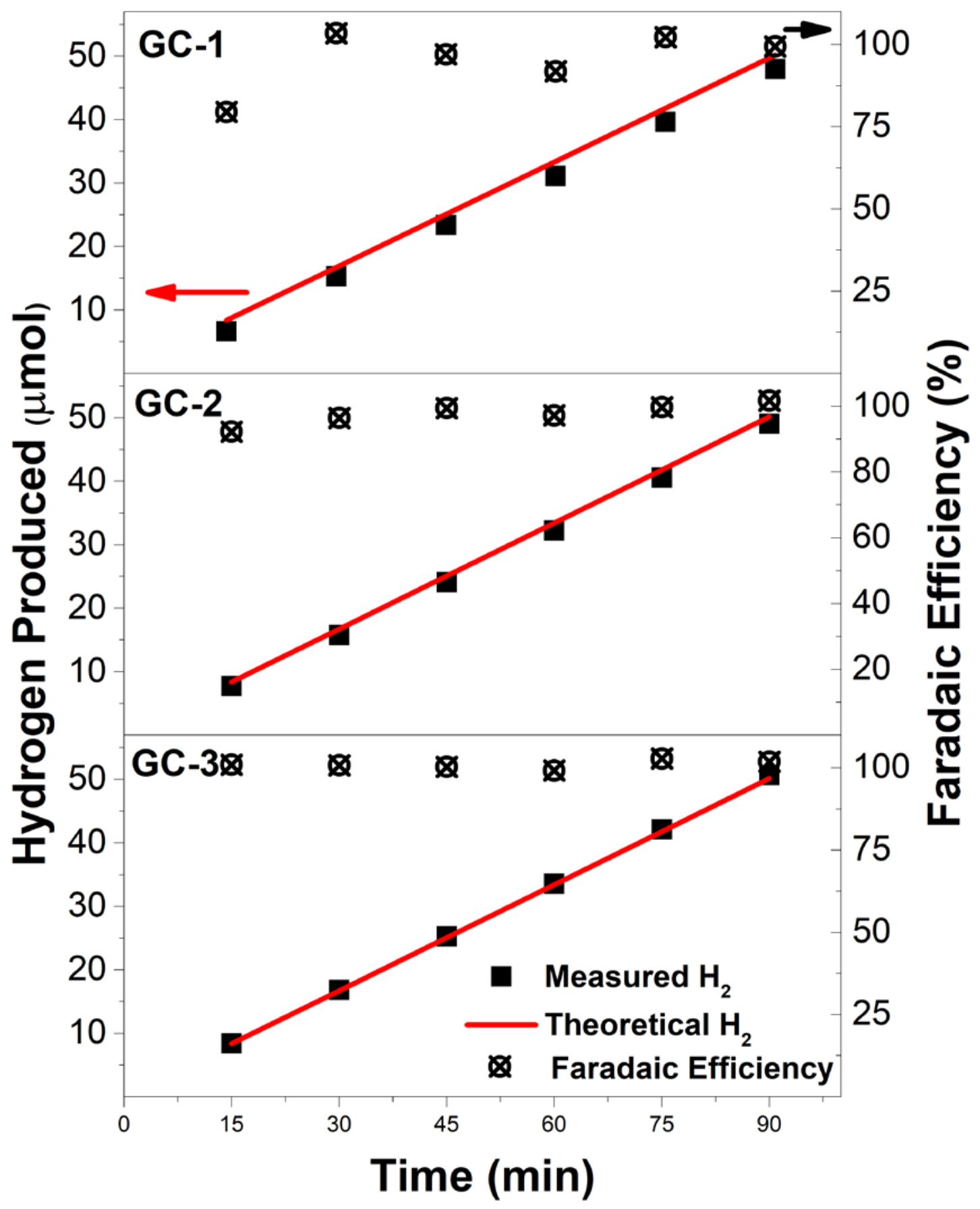

Figure S25. Plot of theoretical hydrogen, measured hydrogen, and Faradaic efficiencies for GC 1 - 3 after cycling to peak activity.

Theoretical $\mathrm{H}_{2}$ is calculated from the coulombs of charge passed during the experiment. Measured $\mathrm{H}_{2}$ was detected via gas chromatography. The Faradaic efficiency was calculating by comparing these two values. GC $\mathbf{1}$ - $\mathbf{3}$ were evaluated after 300, 200, and 300 cycles respectively. 


\section{References}

1. $\quad$ Blower, P. J.; Castle, T. C.; Cowley, A. R.; Dilworth, J. R.; Donnelly, P. S.; Labisbal, E.; Sowrey, F. E.; Teat, S. J.; Went, M. J. Structural trends in copper(II) bis(thiosemicarbazone) radiopharmaceuticals. Dalton Trans. 2003, 4416-4425

2. Johnson, D. ZView: a software program for IES analysis, Version 2.8, Scribner Associates. Inc., Southern Pines, NC 2002, 200

3. Chinarro, E.; Jurado, J.; Figueiredo, F.; Frade, J. Bulk and grain boundary conductivity of $\mathrm{Ca}_{0.97} \mathrm{Ti}_{1-x} \mathrm{Fe}_{x} \mathrm{O}_{3-\delta}$ materials. Solid State Ionics 2003, 160, 161-168

4. Gore, C. M.; White, J. O.; Wachsman, E. D.; Thangadurai, V. Effect of composition and microstructure on electrical properties and $\mathrm{CO}_{2}$ stability of donor-doped, proton conducting $\mathrm{BaCe}_{1-(x+y)} \mathrm{Zr}_{x} \mathrm{Nb}_{y} \mathrm{O}_{3}$. J. Mater. Chem. A 2014, 2, 2363-2373

5. Li, Q.; Thangadurai, V. Synthesis, structure and electrical properties of Mo-doped $\mathrm{CeO}_{2}-$ materials for SOFCs. Fuel Cells 2009, 9, 684-698

6. Li, Q.; Thangadurai, V. A comparative 2 and 4-probe DC and 2-probe AC electrical conductivity of novel co-doped $\mathrm{Ce}_{0.9-\mathrm{x}} \mathrm{RE}_{\mathrm{x}} \mathrm{Mo}_{0.1} \mathrm{O}_{2.1-0.5 \mathrm{x}}(\mathrm{RE}=\mathrm{Y}, \mathrm{Sm}, \mathrm{Gd} ; \mathrm{x}=0.2,0.3)$. J. Mater. Chem. 2010, 20, 7970-7983

7. Hjalmarsson, P.; Søgaard, M.; Mogensen, M. Electrochemical behaviour of $\left(\mathrm{La}_{1-x} \mathrm{Sr}_{x}\right)_{s} \mathrm{Co}_{1-y} \mathrm{Ni}_{y} \mathrm{O}_{3-\delta}$ as porous SOFC cathodes. Solid State Ionics 2009, 180, 1395-1405 